\title{
ACTPol: A polarization-sensitive receiver for the Atacama Cosmology Telescope
}

M. D. Niemack ${ }^{1}$, P. A. R. Ade ${ }^{2}$, J. Aguirre ${ }^{3}$, F. Barrientos ${ }^{4}$, J. A. Beall ${ }^{1}$, J. R. Bond ${ }^{5}$, J. Britton ${ }^{1}$, H. M. Cho ${ }^{1}$, S. Das ${ }^{6}$, M. J. Devlin ${ }^{3}$, S. Dicker ${ }^{3}$, J. Dunkley ${ }^{7}$, R. Dünner ${ }^{4}$, J. W. Fowler ${ }^{8}$, A. Hajian ${ }^{5}$, M. Halpern ${ }^{9}$, M. Hasselfield ${ }^{9}$, G. C. Hilton ${ }^{1}$, M. Hilton ${ }^{10}$, J. Hubmayr ${ }^{1}$, J. P. Hughes ${ }^{11}$, L. Infante ${ }^{4}$, K. D. Irwin ${ }^{1}$, N. Jarosik ${ }^{8}$, J. Klein ${ }^{3}$, A. Kosowsky ${ }^{12}$, T. A. Marriage ${ }^{13}$, J. McMahon ${ }^{14}$, F. Menanteau ${ }^{11}$, K. Moodley ${ }^{10}$, J. P. Nibarger ${ }^{1}$, M. R. Nolta ${ }^{5}$, L. A. Page ${ }^{8}$, B. Partridge ${ }^{15}$, E. D. Reese ${ }^{3}$, J. Sievers ${ }^{5}$, D. N. Spergel ${ }^{13}$, S. T. Staggs ${ }^{8}$, R. Thornton ${ }^{16}$, C. Tucker ${ }^{2}$, E. Wollack ${ }^{17}, \mathrm{~K}$. W. Yoon ${ }^{1}$

${ }^{1}$ National Institute of Standards and Technology, 325 Broadway MC 817.03, Boulder, CO 80305, USA

2 School of Physics and Astronomy, Cardiff Univ., The Parade, Cardiff, Wales, UK CF24 3AA

${ }^{3}$ Department of Physics and Astronomy, Univ. of Pennsylvania, 209 South 33rd Street, Philadelphia, PA, USA 19104

${ }^{4}$ Departamento de Astronomía y Astrofísica, Pontificía Univ. Católica, Casilla 306, Santiago 22, Chile

${ }^{5}$ Canadian Institute for Theoretical Astrophysics, Univ. of Toronto, Toronto, ON, Canada M5S 3H8

${ }^{6}$ Berkeley Center for Cosmological Physics, LBL and Dept. of Physics, Univ. of California, Berkeley, CA, USA 94720

7 Department of Astrophysics, Oxford University, Oxford, UK OX1 3RH

8 Joseph Henry Laboratories of Physics, Jadwin Hall, Princeton University, Princeton, NJ, USA 08544

9 Department of Physics and Astronomy, University of British Columbia, Vancouver, BC, Canada V6T 1Z4

${ }^{10}$ School of Mathematical Sciences, University of KwaZulu-Natal, Durban, 4041, South Africa

${ }^{11}$ Department of Physics and Astronomy, Rutgers, The State University of New Jersey, Piscataway, NJ USA 08854

12 Department of Physics and Astronomy, University of Pittsburgh, Pittsburgh, PA, USA 15260

13 Department of Astrophysical Sciences, Peyton Hall, Princeton University, Princeton, NJ USA 08544

${ }^{14}$ Physics Department, University of Michigan, 450 Church Street, Ann Arbor, Michigan 48109

15 Department of Physics and Astronomy, Haverford College, Haverford, PA, USA 19041

${ }^{16}$ Department of Physics, West Chester University of Pennsylvania, West Chester, PA, USA 19383

17 NASA/Goddard Space Flight Center, Code 553/665, Greenbelt, MD, USA 20771

\begin{abstract}
The six-meter Atacama Cosmology Telescope (ACT) in Chile was built to measure the cosmic microwave background $(\mathrm{CMB})$ at arcminute angular scales. We are building a new polarization sensitive receiver for ACT (ACTPol). ACTPol will characterize the gravitational lensing of the CMB and aims to constrain the sum of the neutrino masses with $\sim 0.05 \mathrm{eV}$ precision, the running of the spectral index of inflation-induced fluctuations, and the primordial helium abundance to better than $1 \%$. Our observing fields will overlap with the SDSS BOSS survey at optical wavelengths, enabling a variety of cross-correlation science, including studies of the growth of cosmic structure from Sunyaev-Zel'dovich observations of clusters of galaxies as well as independent constraints on the sum of the neutrino masses. We describe the science objectives and the initial receiver design.
\end{abstract}

Keywords: B-modes, Cosmic Microwave Background, Cryogenics, Gravitational Lensing, Neutrino Mass, Optical Design, Polarization, Transition-Edge-Sensor Detector Arrays

\section{INTRODUCTION}

The Atacama Cosmology Telescope (ACT) has been used since 2008 to observe temperature anisotropies in the Cosmic Microwave Background (CMB) between wavelengths of one to two millimeters. ${ }^{1}$ These observations led to improved measurements of the CMB temperature anisotropy power spectrum ${ }^{2}$ on arcminute angular scales and detections of galaxy clusters via the Sunyaev-Zel'dovich (SZ) effect. ${ }^{3}$ Multi-frequency data reduction and analysis of point sources, blind SZ detections, and cosmological constraints are underway, while observations with ACT continue.

\footnotetext{
Send correspondence to niemack@nist.gov.
} 

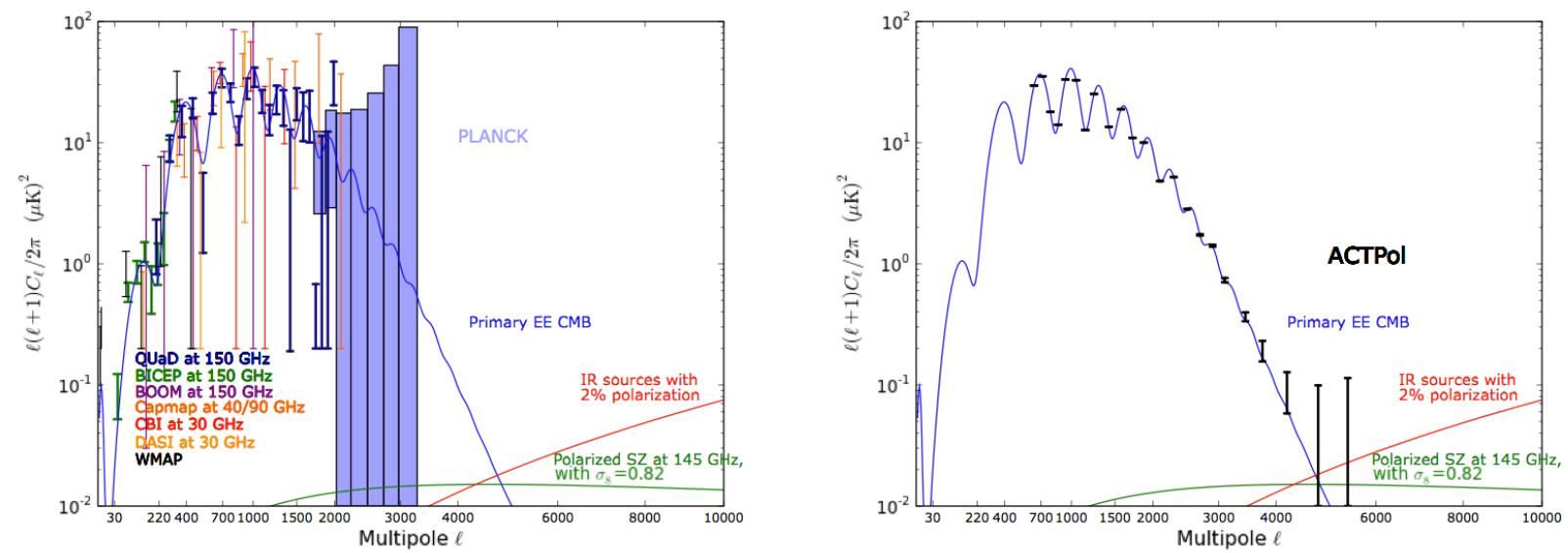

Figure 1. Left: The current state of measurements of the E-mode polarization (EE) power spectrum plotted over the best

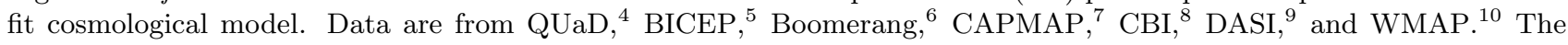
projected Planck error bars (from the "Planck Bluebook") are shown as blue boxes. The foregrounds are conservative estimates for infrared point sources and a $1 \%$ net polarization of the SZ effect. Right: Projection of the ACTPol errors for the deep fields $\left(150 \mathrm{deg}^{2}\right)$ based on the target instrument sensitivity described in $\$ 5$ and 1300 hours of observations by use of the Knox formula, $\frac{11}{11}$ which ignores foregrounds, systematic errors, and extra noise from the atmosphere. We do not project errors at $\ell<500$ because the Knox formula is inaccurate at large scales in the presence of atmospheric fluctuations.

We are developing a new dual-frequency $(150 \mathrm{GHz}$ and $220 \mathrm{GHz})$ polarization sensitive receiver (ACTPol) to be deployed on ACT in 2013. In addition to being polarization sensitive, it is projected to substantially improve upon the temperature sensitivity of the current receiver. These improvements will enable an expansion of the current ACT science goals, which are based on a range of observations that go well beyond the CMB power spectra, and combine to provide a built-in set of cross-checks. ACTPol will focus on a four-pronged science program:

1. Measure the intrinsic temperature and polarization anisotropy at high-multipoles $(\ell)$ to probe the spectral index of inflation, the primordial helium abundance, and neutrino properties.

2. Measure the gravitational lensing of the $\mathrm{CMB}$ in temperature and polarization to constrain early dark energy and the sum of the neutrino masses.

3. Correlate and compare the CMB with lower redshift optical, infrared, and X-ray surveys to achieve a variety of science goals, including independent constraints on the sum of the neutrino masses.

4. Find clusters of galaxies through their Sunyaev-Zel'dovich effect and combine them with other surveys to study the growth of structure.

These four measurement objectives and science goals are described in more detail in $\$ 2$ where we also present predicted results based on the target ACTPol performance. The ACTPol observing strategy, foreground emission, and calibration are discussed in $\$ 3$, the ACT design is reviewed in $\$ 4$, and an initial ACTPol receiver design and sensitivity forecast are introduced in $\$ 5$.

\section{SCIENCE GOALS}

\subsection{Measure the Intrinsic Temperature and Polarization Anisotropies at $\ell \gtrsim 2000$}

The temperature anisotropy may be divided roughly into large $(\ell \lesssim 2000)$ and small $(\ell \gtrsim 2000)$ angular scales. At large angular scales accessible to the WMAP and Planck ${ }^{\text {F }}$ satellite telescopes, the anisotropy is a direct probe

*Planck uncertainties from: http://www.rssd.esa.int/Planck 

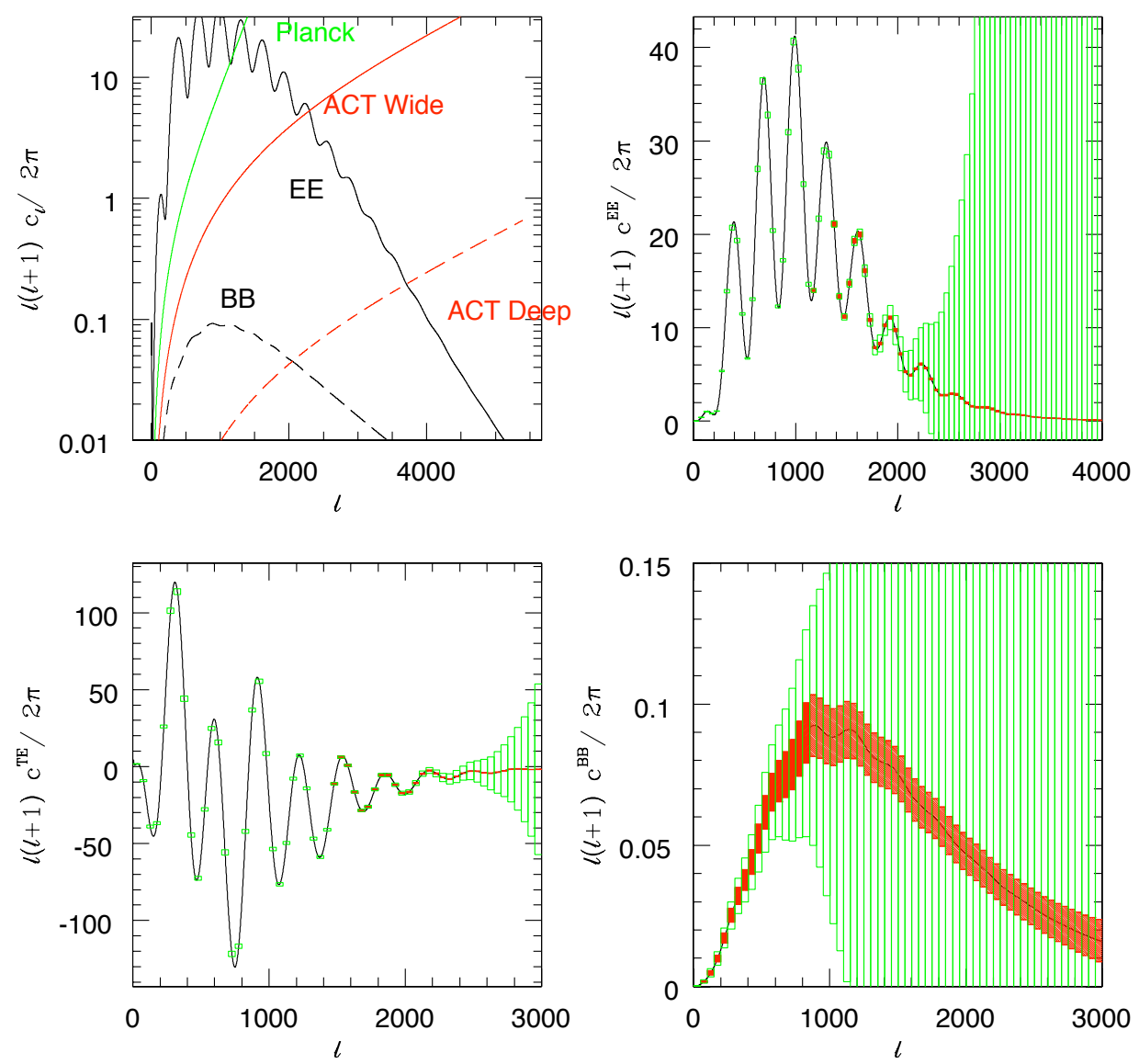

Figure 2. The upper left panel shows the statistical noise per multipole for Planck, ACTPol Wide, and ACTPol Deep (\$3) and the predicted EE and BB power spectra. The BB spectrum is due to lensing. The other three panels show the $\mathrm{EE}, \mathrm{BB}$, and TE spectra with target ACTPol errors (filled red boxes, which are small on a linear scale except in the BB spectrum; Figure 1 shows the EE spectrum on a logarithmic scale) and Planck errors (open green boxes). At low $\ell$, when the Planck errors are smaller, only they (open green boxes) are shown. In the BB spectrum the red boxes at $\ell<850$ are for the ACTPol wide survey (3), while the red boxes at $\ell>850$ are for the ACTPol deep survey. The latter three panels are binned with $\Delta \ell=50$. Atmospheric contamination may preclude ACTPol measurements below $\ell \sim 500$; however, the atmosphere is not polarized at these frequencies, which could enable polarization measurements at lower $\ell$ than temperature measurements. The y-axes are in $\mu \mathrm{K}^{2}$.

of the response of the CMB to perturbations laid down in the early Universe as seen at a redshift of $z \sim 1000$. At smaller angular scales nonlinear physics (structure formation) dominates, and the foreground contamination by point sources such as radio and starforming galaxies becomes significant. Measuring the transition between these two regimes in both temperature and polarization will improve constraints on the standard cosmological model.

Our planned ACTPol surveys ( $\$ 3$ are designed to continue characterizing the temperature (TT) foregrounds out to $\ell \sim 8,000$ and to measure the E-mode polarization (EE), temperature to E-mode (TE), and B-mode polarization $(\mathrm{BB})$ power spectra with high signal-to-noise ratio (see Figures 1 and 2 ) out to $\ell \gtrsim 3000$. These data will allow us to probe early-Universe physics, reduce errors on cosmological parameters, measure the number of neutrino species, and determine the helium abundance (Table 1). We address the science related to the various power spectra below. Although the results are derived from the combined analysis of all spectra, we concentrate on TT and EE in this section, and focus on BB when we discuss lensing (\$2.2). 


\begin{tabular}{|c|c|c|c|c|}
\hline \hline & WMAP5 & WMAP9 + ACTPol & Planck & Planck + ACTPol \\
\hline$\Omega_{b} h^{2}$ & $6 \times 10^{-4}$ & $1.9 \times 10^{-4}$ & $2 \times 10^{-4}$ & $1.3 \times 10^{-4}$ \\
$\Omega_{m} h^{2}$ & $6 \times 10^{-3}$ & $3.7 \times 10^{-3}$ & $2.2 \times 10^{-3}$ & $1.7 \times 10^{-3}$ \\
$n_{s}$ & 0.014 & 0.008 & 0.007 & 0.006 \\
$m_{\nu}$ & - & 0.15 & 0.1 & 0.06 \\
$\tau$ & 0.017 & 0.010 & 0.004 & 0.004 \\
$Y_{H e}$ & - & 0.007 & 0.01 & 0.005 \\
\hline \hline
\end{tabular}

Table 1. This table shows projected $68 \%$ confidence interval constraints in an 8-parameter model (baryon density, matter density, $n_{s}$, neutrino mass (in eV), optical depth, helium abundance, Hubble expansion factor, amplitude) from the ACTPol data with WMAP and Planck based on a Fisher matrix analysis without systematic errors. For a 7-parameter model (baryon density, matter density, $n_{s}$, optical depth, Hubble expansion factor, amplitude, $\alpha$ ) with Planck+ACTPol the constraint on $\alpha$ is 0.004 . For comparison, current constraints from WMAP5 are shown in which only 6 parameters are fit. The WMAP data set after 9 years is named WMAP9.

Inflation and early Universe physics. The small-scale temperature anisotropy is important for testing models of the early Universe. The most favored model is inflation,,$\frac{12}{15}$ though there are variants and alternatives. Assuming adiabatic and Gaussian fluctuations, there are three early-Universe parameters one can get directly from the CMB: the scalar spectral index, $n_{s}$, or overall slope of the angular power spectrum after removing the acoustic oscillations, $; \frac{16 \sqrt{20}}{12}$ the running of the spectral index with wavenumber $k$ or $\left.\alpha \equiv d n_{s} / d \ln k, 21\right]$ and the tensor to scalar ratio, $r$, which describes the degree to which the Universe is suffused with a cosmic background of gravitational waves. Of these, ACTPol will focus on constraining $n_{s}$ and $\alpha$ by measuring the $\ell>500$ E-mode polarization. In principle ACTPol can measure the low- $\ell$ gravitational wave B-mode spectum, but we believe the low- $\ell$ polarization measurements are best done with optimized dedicated instruments. Although inflationrelated tensor modes are directly manifest at low $\ell$, high- $\ell$ measurements can also constrain them through their covariance with other parameters, most notably $n_{s}$.

All indications are that $n_{s}$ is slightly less than unity, which is in line with the predictions of simple models of inflation, $n_{s} \approx 0.95$. Recent work by the QUaD collaboration ${ }^{4}$ gives an example of what can be done by adding just $\ell<2000 \mathrm{CMB}$ data $(\mathrm{ACBAR}+\mathrm{QUaD})$ to the WMAP results. They find $n_{s}=0.96 \pm 0.012$ and $\alpha=-0.028 \pm 0.018$.

High-resolution and high-sensitivity E-mode measurements offer a new way to measure $n_{s}$ and $\alpha$. Even though the EE spectrum at $\ell \sim 3000$ is one-sixth as large in temperature as the TT spectrum, the polarized point source contamination is far less (see 33 . Thus, EE measurements offer a relatively point-source-contamination-free way to measure the key inflation parameters, $n_{s}$ and $\alpha$. Through the EE spectrum, ACTPol should be able to directly probe the primordial power spectrum out to $\ell \sim 3500$.

Non-Gaussianity. In the standard model of cosmology, the fluctuations are Gaussian. It is widely believed that if the model is incomplete or incorrect the first hints will come through the detection of non-Gaussianity ${ }^{22}$ To date there are only upper limits on non-Gaussianity. As one example of physics beyond the standard model, we studied the detectability of cosmic strings through their imprint on the $\mathrm{CMB}^{23}$ in the presence of secondary anisotropies and found that ACTPol could place improved limits of $\mathrm{G} \mu<10^{-7}$ (in Planck units) on the string tension.

Helium abundance, neutrinos, relativistic species, and the standard model. The high- $\ell$ EE spectrum is sensitive to the primordial helium abundance because the associated high- $\ell$ modes enter the horizon before the helium fully recombines $24 \sqrt[25]{25}$ We aim to measure the primordial helium abundance, $Y_{H e}$, to better than $1 \%$, independent of systematic effects in the astronomical determination of the abundance, which will allow precise measurements of the baryon density and thus the spectral index

\footnotetext{
${ }^{\dagger}$ Currently, the parameter with the greatest degeneracy with $n_{s}$ is $\Omega_{b} h^{2}$. The optical depth, $\tau$, is known well enough that the $n_{s}-\tau$ degeneracy is broken.
} 
in Table 1, should have the same errors as those quoted for measurements of primordial helium abundance from white dwarfs.26 The combination of precise measurements of helium abundances and $\Omega_{b} h^{2}$ will enable powerful constraints on neutrino properties and early-Universe physics. ACTPol aims to constrain the sum of the neutrino masses in multiple ways, with projected accuracies between 0.05 and $0.07 \mathrm{eV}(\$ 2.2)$, a substantial improvement on the recent WMAP constraints of $<1.3 \mathrm{eV} \stackrel{27}{ }$ Measurements of atmospheric neutrino oscillations

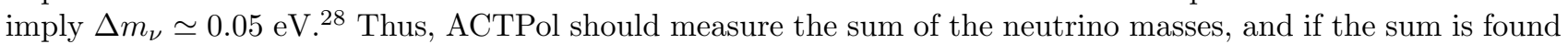
to be $\lesssim 0.1 \mathrm{eV}$, this measurement would rule out the possibility of an inverted neutrino mass hierarchy 29

A high- $\ell$ measurement of the EE spectrum will allow significantly improved constraints on the effective number of neutrino species, which in the standard model is $N_{\text {eff }}=3.04$. Additional relativistic species (e.g., gravitons, Goldstone bosons) could contribute to the radiation density or non-standard neutrino interactions could exist that would alter $N_{\text {eff }}$, either of which would induce a distinct phase shift in the positions of the acoustic peaks. ${ }^{30}$ Current constraints from WMAP and other cosmological data give $N_{\text {eff }}=4.3 \pm 0.9 !^{31}$ ACTPol will probe physics beyond the standard model by improving this constraint by more than a factor of two.

\subsection{CMB Lensing and B-mode Polarization}

At high $\ell$, the B-mode polarization is produced by gravitational lensing of the E-modes ${ }^{32}$ The lensing power spectrum can give an excellent measure of the neutrino mass sum, spatial curvature, and "early dark energy" ${ }^{33}$ CMB lensing provides the best way to study the nature of dark energy at early times, because the lensing kernel probes a wide range of redshifts that peaks at $z \sim 2-4$, while low-redshift cosmological probes, including optical galaxy lensing measurements, are sensitive to cosmology at $z \lesssim 1$. ACTPol is designed to measure the lensing in both temperature and polarization.

ACTPol's measurements of the CMB lensing power spectrum can provide an accurate measurement of the amplitude of matter fluctuations at $z \sim 2-4$. This measurement, in turn, can provide a sensitive determination of neutrino mass of $\sim 0.07 \mathrm{eV}$, close to the observed mass splitting (see Table 1); and complement weak lensing surveys that trace the mass fluctuations in mass sheets at $z \lesssim 1$. By providing a high- $z$ anchor for planned dark energy surveys, ACTPol will improve measurements of dark-energy effects on structure growth.

ACTPol should be able to measure lensing to a high enough precision to "delens", or reconstruct the true CMB fluctuation power spectrum. It could thus improve the $\mathrm{S} / \mathrm{N}$ on large-angular-scale, primordial B-mode measurements by a factor of $\sim 1.8^{34}$ By combining CMB lensing data with measurements from the surface of last scattering to break the geometric degeneracy ${ }^{355}$ WMAP9 + ACTPol should be able to measure the curvature of the universe to better than $0.5 \%$ by use of CMB data alone.

By cross-correlating the lensing of the CMB with the variance in the fluctuations in the Ly $\alpha$ forest, we will directly probe the relationship between matter and gas in the Ly $\alpha$ forest. Vallinotto et al [36 $^{36}$ estimate a S/N $=20$ cross-correlation signal for ACTPol with BOSS. This measurement leads to a $5 \%$ determination of the amplitude of the Ly $\alpha$ power spectrum, which leads to another measurement of neutrino mass with $\sigma_{\nu} \sim 0.05 \mathrm{eV}$.

The combination of WMAP and the SDSS 7 Luminous Red Galaxy (LRG) survey $(z \sim 0.3)$ has already placed powerful limits on cosmological parameters 3738 The BOSS\$ LRG sample will extend the range, providing an accurate determination of the galaxy power spectrum for $z=0.3-0.7$. Acquaviva et al ${ }^{39}$ have estimated a $\mathrm{S} / \mathrm{N}$ of 11, 25 and 40 for cross correlations of ACTPol with SDSS DR7 LRG, BOSS low-redshift LRG, and BOSS LRG samples. This translates to another independent measurement of neutrino mass with $\sigma_{\nu} \sim 0.05 \mathrm{eV}$, an improvement over the ACTPol-only constraint, and provides yet another method of assessing the effects of dark energy on structure growth. 39

\subsection{SZ Clusters, an Unbiased Search}

One well studied perturbation of the CMB at small angular scales is the Sunyaev-Zel'dovich (SZ) effect, in which the hot electrons $\left(10^{7} \mathrm{~K}-10^{8} \mathrm{~K}, \sim 5 \mathrm{keV}\right)$ in galaxy clusters reveal their presence by scattering the CMB with a characteristic frequency signature ${ }^{40}$ The process of finding hundreds to thousands of clusters in large blind and unbiased surveys has already been started by the SPT ${ }^{41}$ and $\mathrm{ACT}^{3}$ groups. These are the two best telescopes

\footnotetext{
${ }^{\ddagger}$ SDSS: The Sloan Digital Sky Survey. http://www.sdss.org/

${ }^{\S}$ BOSS: The Baryonic Oscillation Spectroscopic Survey. http://cosmology.lbl.gov/BOSS/
} 
in the world for these studies. ACT enjoys the ability to overlap with many optical and radio observations of clusters made by telescopes in both the northern and southern hemispheres. We aim to find $\sim 1000$ clusters in the ACTPol surveys $42[43$

With the SZ detected clusters in hand, cluster redshifts are found through optical follow-up. The mass selection function is determined with a combination of SZ, optical (cluster-member spectroscopy and weak lensing), and X-ray measurements for a subset of the clusters, allowing mass-observable relations to be used for the larger sample. The masses and catalogs enable studies of the number distribution, $d N / d z$ or $d N(>M) / d M$, which is exponentially sensitive to the dark matter and dark energy densities and serves as a potent test for the amplitude of matter fluctuations, $\sigma_{8}$, and the equation of state of dark energy, $w \underline{44}$ The combination of wide and deep SZ surveys $(\$ 3$ helps to break cosmological parameter degeneracies thus improving constraints. $\underline{46}$

\subsection{Additional Correlations with Low Redshift Data}

As the Universe evolves, galaxies and clusters of galaxies emerge from the primordial plasma and leave their imprint on the CMB. The CMB can be related to the foreground objects through scattering (SZ, kSZ, and reionization effects) or through gravitational lensing as described above. Additionally, by correlating CMB observations with galaxy velocity fields inferred from spectroscopic data, one may search for the "missing baryons" in the outskirts of clusters 47 In another example, ACTPol cross-correlation with massive galaxies has the potential to measure the energy feedback from supermassive black holes, which heats the surrounding intergalactic medium and creates a small-scale SZ distortion. $\frac{48}{}$ It may even be possible to detect the Kamionkowski-Loeb effect ${ }^{49}$ to see whether the quadrupole seen by observers at $z \simeq 0.3-0.7$ is similar in character to the one we observe today. The various phenomena are distinguishable through their specific correlations and spatial distributions. The cross correlations may be done with radio, infrared, optical, and X-ray surveys, and through gravitational lensing as discussed above.

\section{OBSERVING STRATEGY}

One of the great benefits of ACT is its location. Some of the ACT science requires that we use data from external surveys (e.g., to measure cluster redshifts, X-ray masses, and lensing masses for the SZ surveys). In other cases, the cross-correlations open up new avenues of investigation and provide alternative methods for achieving the science goals (e.g., measuring the sum of the neutrino masses). The location of ACT enables observations of the same regions of sky covered by many other surveys. Figure 3 shows the accessible coverage along with the coverage of some overlapping surveys. With its elevation range of $40^{\circ}$ to $55^{\circ}$ for science observations and latitude of $-23^{\circ}$, ACT can observe over $25,000 \mathrm{deg}^{2}$ in $-73^{\circ}<\delta<27^{\circ}$.

Our observing strategy is based on a balance of sensitivity requirements, the need to calibrate to WMAP and Planck, and the desire to optimize the lensing signal and the overlap with other surveys while also maximizing the cluster science. The current temperature observations focus on two regions. The "equatorial strip" overlaps the SDSS Stripe 82 and part of the BOSS region, and somewhat less deeply the GAMA fields It It is one of the best studied regions of sky. The other, the "southern strip," covers $-55^{\circ}<\delta<-49^{\circ}$ and overlaps the Blanco Cosmology Survey (BCS 50 ) fields (Figure 3). The planned ACTPol observing strategy builds on this basis of multi-frequency coverage but implements significant contrast between "deep" and "wide" coverage.

\subsection{Sky Coverage and Overlap}

The first three ACTPol observing seasons will be split between a wide survey covering $4000 \mathrm{deg}^{2}$ to a target sensitivity of $20 \mu \mathrm{K} / \mathrm{arcmin}^{2}$ in temperature and $28 \mu \mathrm{K} / \mathrm{arcmin}^{2}$ in polarization at $150 \mathrm{GHz}$ (ACTPol Wide) and a deep survey that covers five $2^{\circ} \times 15^{\circ}$ regions with a target sensitivity of $3 \mu \mathrm{K} / \operatorname{arcmin}^{2}$ in temperature and $4 \mu \mathrm{K} / \operatorname{arcmin}^{2}$ in polarization at $150 \mathrm{GHz}$ (ACTPol Deep).

The wide fields are designed for maximal overlap with rich multi-wavelength imaging data and spectroscopic data, enabling unique multi-wavelength science. With the wide fields we measure the growth rate of structure, the neutrino mass, curvature, and early-time dark energy, as discussed in $\$ 2$.

\footnotetext{
IThe GAMA project, http://www.eso.org/jiske/gama/
} 


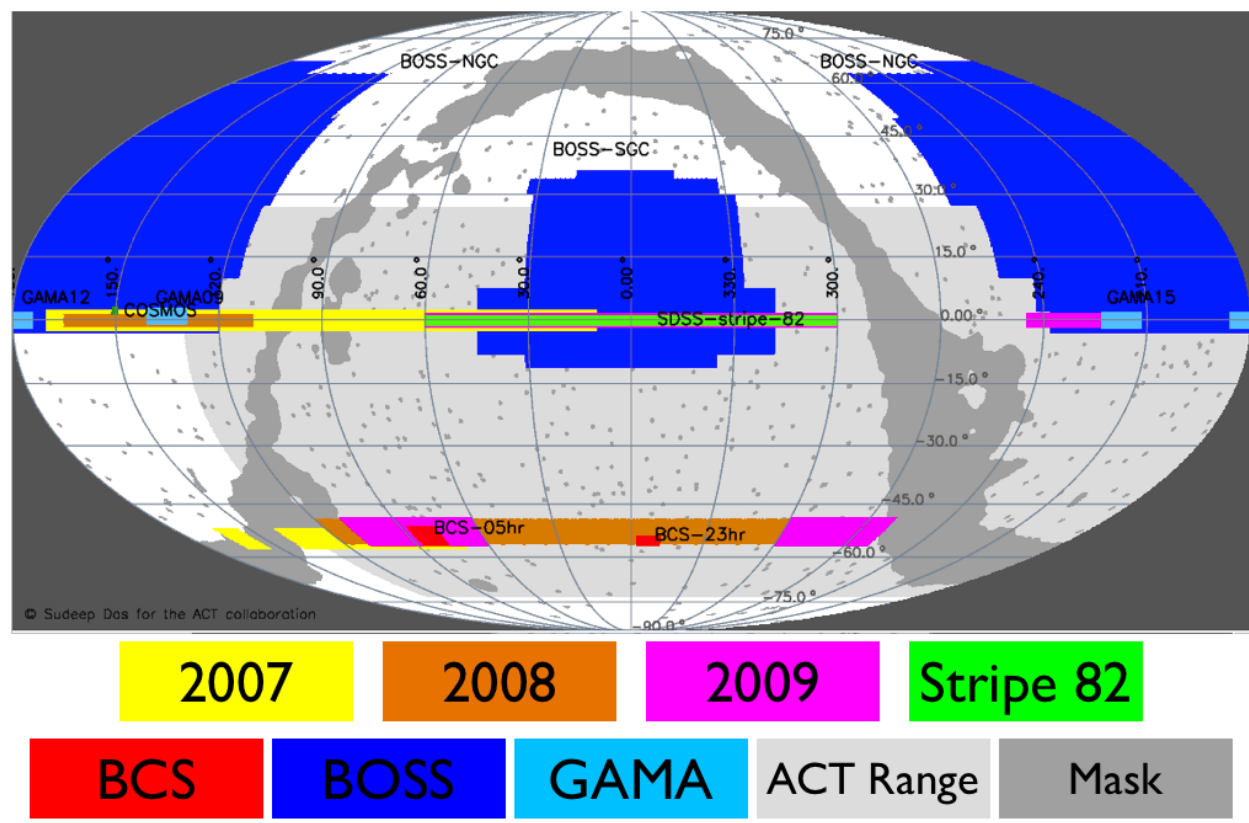

Figure 3. Equal-area projection of the ACT sky coverage in celestial coordinates. The darker gray Galaxy and spots correspond to the WMAP galactic and point source mask. The light gray shows the $\sim 25,000 \mathrm{deg}^{2}$ accessible to ACT. The colored areas indicate observations by ACT during 2007-9 (yellow, orange and pink), BCS (red), COSMOS, SDSS stripe 82 (green), BOSS (dark blue), and GAMA (light blue) surveys. The 2009 ACT coverage overlaps with the SDSS equatorial stripe. On the equator, ACT has covered $800 \mathrm{deg}^{2}, 220 \mathrm{deg}^{2}$, and $440 \mathrm{deg}^{2}$ in 2007-9 respectively. In the southern strip, ACT has covered $280 \mathrm{deg}^{2}, 800 \mathrm{deg}^{2}$, and $450 \mathrm{deg}^{2}$ in 2007-9, respectively. The total BOSS coverage is $7500 \mathrm{deg}^{2}$ in the NGC and $3100 \mathrm{deg}^{2}$ in the SGC, of which ACT can overlap $2300 \mathrm{deg}^{2}$ and $2700 \mathrm{deg}^{2}$, respectively. The region between $130^{\circ}<R A<180^{\circ}$ corresponds to the area that is difficult to observe with both rising and setting scans, since we typically go offline from Dec 15-Apr 1 due to poor observing conditions. This region is accessible, as can be seen in our 2007-8 equatorial coverage; it is just not optimal.

The ACTPol Wide field will overlap with the BOSS survey. BOSS will carry out a redshift survey of 1.5 million LRGs over 10,000 $\mathrm{deg}^{2}$ and 160,000 QSOs over an 8,000 $\mathrm{deg}^{2}$ footprintll. ACTPol is projected to detect $\sim 1000$ clusters with $M_{\odot}>5 \times 10^{14}$ in this field 42433 These clusters will have a median redshift of $\sim 0.4$, and so should significantly overlap with the SDSS cluster catalogs.

In addition to overlapping with BOSS, we are collaborating on a project to conduct a wide $\left(2000 \mathrm{deg}^{2}\right.$ $8000 \mathrm{deg}^{2}$ ) optical lensing survey of the equatorial fields with HyperSuprime Camera (HSC), a $1.5^{\circ}$ FOV camera on the Subaru telescope. The data will be optimized for weak lensing and will include measurements in the near-infrared $\mathrm{Y}$ band to detect $z>1$ galaxies.

The deep regions are designed especially to optimize the signal-to-noise on the polarization and temperature and simultaneously allow for detailed studies of cluster masses. They will be spaced roughly 5 hours apart so that there are always two deep fields available for observation. We have tentatively selected the fields to overlap regions with deep optical, infrared, and X-ray observations (XMM-LSS), and the Herschel Multi-tiered Extragalactic Survey (HERMES).

HSC also plans a deep $300 \mathrm{deg}^{2}$ survey in five optical bands $(g, r, i, z, y)$ and has agreed to work to maximize the overlap between the ACT and HSC survey regions. When HSC has mapped a significant fraction of the ACTPol Deep survey, we will be able to identify photometric counterparts for the observed clusters and measure their masses.

\footnotetext{
"Additionally, SDSS has 600,000 quasars with photometric redshifts, ${ }^{[51]}$ and 15,000 MaxBCG clusters, ${ }^{[52]}$ and NVSS has identified 400,000 1.4 GHz sources in the ACTPol Wide field.
} 


\subsection{Foreground Emission}

Although interesting in its own right, foreground emission from diffuse sources such as dust and synchrotron emission in our Galaxy and from radio/IR point sources outside the Galaxy complicates some cosmological analyses.

For temperature anisotropy, in the regions of the sky selected by ACT, $150 \mathrm{GHz}$ emission is contributed by both flat-spectrum radio sources (generally the brighter $150 \mathrm{GHz}$ sources) and by IR sources (generally fainter but more numerous 5 ). Our top-level approach to reduce point-source contamination in ACTPol images is to clean the $150 \mathrm{GHz}$ data by use of the $220 \mathrm{GHz}$ data and surveys of radio sources conducted at lower frequencies (such as the $20 \mathrm{GHz}$ survey of Murphy et al ${ }^{[55}$ ). Ultimately the degree of cleaning will limit what can be learned at $150 \mathrm{GHz}$ from total intensity (i.e., unpolarized) maps. The point sources should not have a large effect in the cross-correlation studies.

While foregrounds dominate the CMB temperature fluctuations at $\ell>2500$, CMB polarization fluctuations should dominate foregrounds sources out to $\ell \sim 5000$ (Figure 1). The dominant foreground source above $\sim 100 \mathrm{GHz}$ is expected to be dusty galaxies, which are only weakly polarized (1\%-2\%) 56 wh wile the CMB is highly polarized $(17 \%)$ at $l>1000$. The cleanest frequency for polarization is near $150 \mathrm{GHz} \cdot \frac{58}{5}$ Synchrotron emission is a significant contaminant even at $90 \mathrm{GHz}$ and is much more diffuse than the dust, which concentrates in the galactic plane ${ }^{59}$ Radio point sources are polarized at the $1 \%-5 \%$ leve $\frac{\sqrt{60}}{6}$ and have already been cleaned from the ACT maps to a depth sufficient for a polarization analysis. Smith et al ${ }^{34}$ report that the impact of point sources on lensing is negligible.

\subsection{Calibration}

As the CMB field matures, a connected set of calibrations is developing. WMAP has calibrated the CMB to $0.2 \%$ accuracy. Calibration of the planets useful to ACT (Mars, Saturn, Uranus) at the few-percent level is well underway 61 For polarization angle calibration, the primary method for ACTPol will also be to map astrophysical sources that have been characterized by other instruments. For example, the polarization angle of Tau-A was measured by WMAP to better than $1^{\circ}{ }^{62}$ Sub-orbital instruments and Planck will continue this process, improving the temperature calibrations and measuring polarization angles with higher precision.

\section{THE ATACAMA COSMOLOGY TELESCOPE}

\subsection{Telescope Overview}

ACT is an off-axis Gregorian telescope with a six-meter projected aperture primary mirror and is located on the Atacama Plateau, Chile at 5190 meters elevation. The size of the primary mirror was driven by the requirement to obtain arcminute resolution at the ACT frequencies. The off-axis Gregorian configuration of the primary and two-meter secondary mirrors provides an unobstructed image of the sky. A detailed description of how the telescope and receiver optical parameters were optimized is presented in Fowler et al ${ }^{1}$ The current receiver on ACT is the Millimeter Bolometer Array Camera (MBAC) $\sqrt[63]{64}$ which includes three bolometer arrays operating at $148 \mathrm{GHz}, 215 \mathrm{GHz}$, and $277 \mathrm{GHz} \frac{65] 66}{66}$ The telescope control systems and mechanical performance are described in Switzer et al ${ }^{[67}$ and Hincks et al. ${ }^{68}$

\subsection{Scan Strategy}

We scan the sky to separate the effects of the atmosphere and instrumental drift from the celestial signal. For $\mathrm{ACT}$, we scan the entire 40 metric ton upper structure $\pm 3.5^{\circ}$ in azimuth while holding the elevation fixed (typically at $50.5^{\circ}$ ). We move the telescope beam on the sky on timescales faster than the $1 / f$ knee of the low frequency noise but more slowly than the time constants of the detectors. Scans are done at two positions east and west of an arc between the south celestial pole (SCP) and the zenith. As the sky rotates, an annulus around the SCP is mapped out.

This observing strategy has several benefits for observations from the ground. First, changing the amount of air mass affects the gain of the system from atmospheric absorption. Keeping the elevation fixed thus reduces scan-synchronous atmospheric signals. Second, by performing both east and west scans, the mapped annulus 


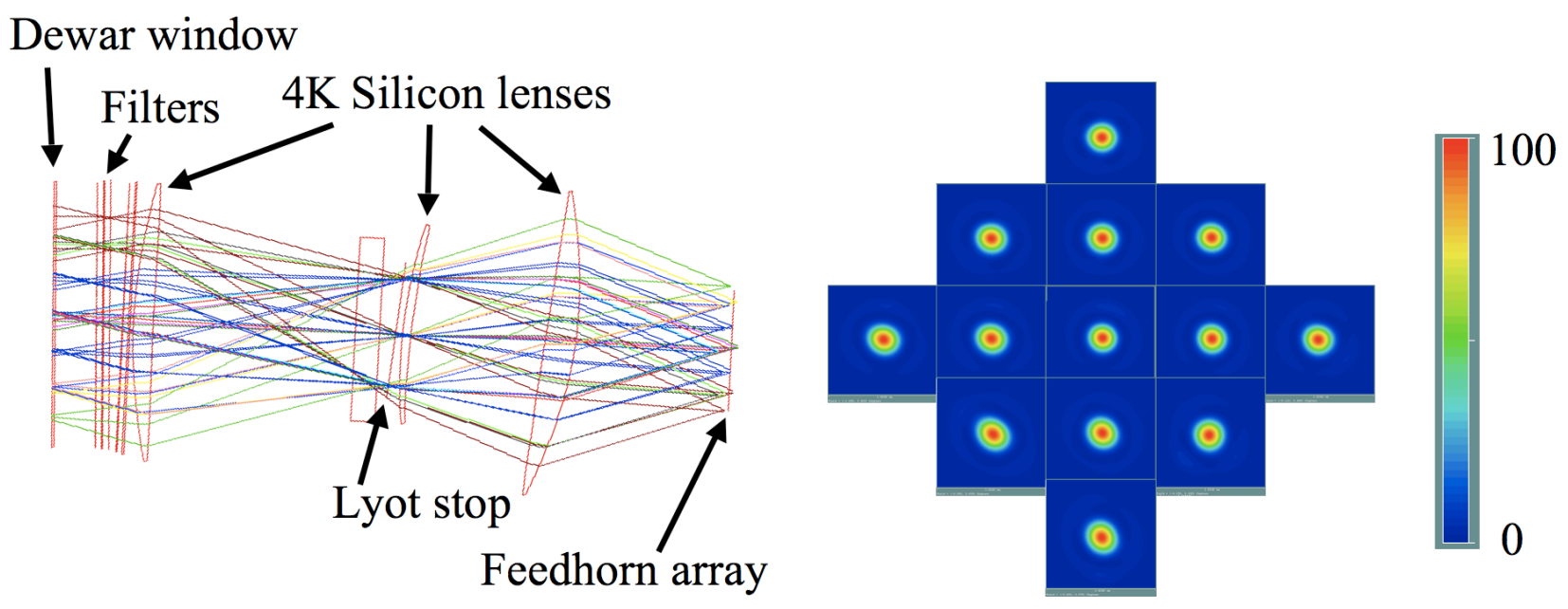

Figure 4. Left: Ray-traced optical design of one of the ACTPol $150 \mathrm{GHz}$ cameras. The Gregorian focus is visible near the dewar window at the left. For scale, the dewar window as drawn is $32 \mathrm{~cm}$ diameter. Three silicon lenses reimage the focus onto a feedhorn array. The Lyot stop is at a primary mirror image and is used to define the primary illumination. The telecentricity is apparent since the chief rays for different field points are parallel to each other and perpendicular to the feedhorn array focal plane. This design achieves Strehl ratios greater than 0.95 across the nearly one degree diameter focal plane. Right: Point spread functions (PSF) calculated for detector positions that cover the entire $150 \mathrm{GHz}$ field of view (e.g., the edge PSFs shown are at the edge of the circular array). The PSF full-width-half-maxima are $\sim 1.4$ arcmin. This design exhibits minimal beam ellipticity or asymmetry.

will be observed in two different cross-linked orientations. Cross-linking has been shown to be highly beneficial for the removal of scanning-induced systematic effects such as striping from the maps $\frac{69}{70}$ Finally, by moving the entire upper structure of the telescope, including the primary, secondary, and receiver, the detectors are constantly looking through the same optical chain. Scanning with the complete optical system on the sky (as opposed to using an optical chopping mirror) avoids many scan-synchronous signals that could potentially arise from changing the optical path such as beam shape, mirror emission, and ground pick-up.

\section{THE ACTPOL RECEIVER}

The ACTPol receiver is a cryogenic instrument designed to measure both the intensity and polarization of radiation between one millimeter and three millimeters in wavelength. Here we describe the coupling optics, detectors, and cryogenics for the receiver.

\subsection{ACTPol Optics Design}

The ACTPol optics are optimized for use with new polarization-sensitive detector arrays being developed by the Truce collaboration ${ }^{* *}$. These arrays use single-moded corrugated feedhorns to couple radiation to the detectors (55.2). The refractive optics are designed to maximize the optical throughput onto three detector arrays by use of three independent optics tubes. Two of the arrays will observe at $150 \mathrm{GHz}$ and the third at $220 \mathrm{GHz}$ (with the possibility of a $90 \mathrm{GHz}$ replacement array). Each optics tube uses three silicon lenses to reimage the Gregorian focus and to generate a Lyot stop, or image of the primary mirror (Figure 4). The optical throughput is limited by the maximum available size of the filters $\left(\sim 30 \mathrm{~cm}\right.$ diameter $\left.{ }^{71}\right)$, which results in a $\sim 1^{\circ}$ diameter field-of-view (FOV) that is well matched to the detector arrays. The resulting $150 \mathrm{GHz}$ feedhorn aperture is $\sim 1.5 F \lambda$, where $F \approx 1.4$ is the focal ratio and $\lambda$ is the wavelength. This spacing results in $\sim 4 \mathrm{~dB}$ edge taper at the Lyot stop, or a feedhorn spillover efficiency of $\sim 0.7$ through the stop.

\footnotetext{
${ }^{* *}$ The Truce detector development collaboration is composed of members at NIST, University of Chicago, University of Colorado, University of Michigan, NASA GSFC, and Princeton University: http://casa.colorado.edu/ñenninjw/TRUCE/TRUCE.html
} 

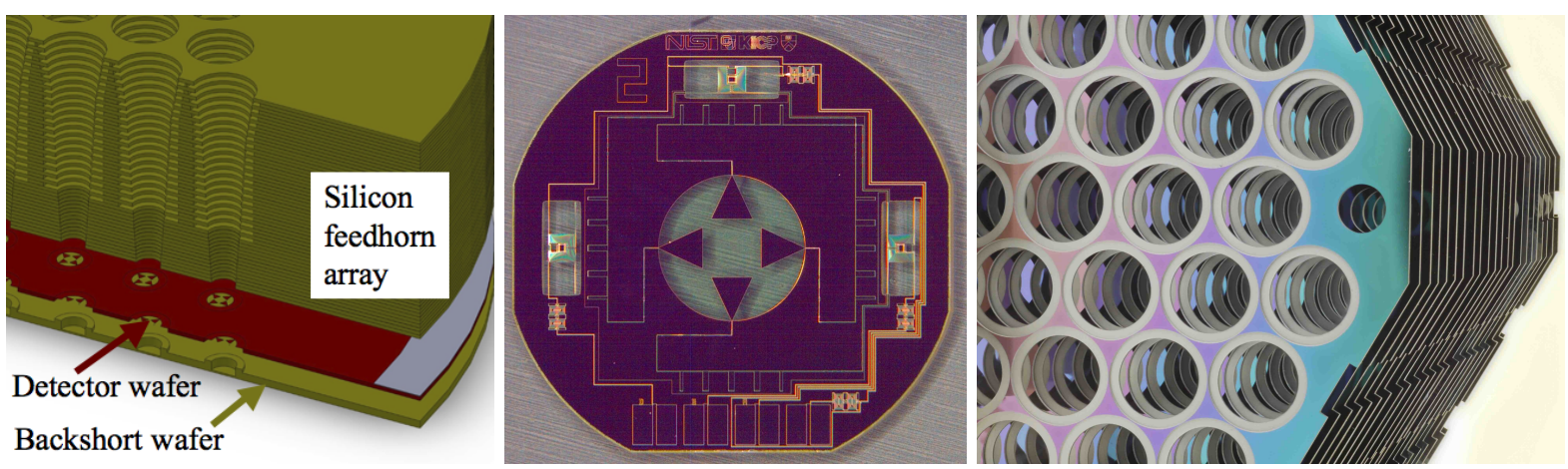

Figure 5. Left: Cutaway view of a silicon feedhorn coupled polarimeter array design. The spacing between the feedhorns is $5.3 \mathrm{~mm}$. The detectors and backshort wafers have been offset from the feedhorn array for clarity. Middle: Photograph of a single prototype polarimeter that is $5 \mathrm{~mm}$ diameter. The polarimeter design and performance have been described in detail. ${ }^{74}$ Individual polarimeters like this will be used by the Atacama B-mode Search, 79 and a monolithic polarimeter array with silicon feedhorns will be used by SPTPol ${ }^{80}$ Right: Etched silicon wafers for a prototype corrugated silicon feedhorn array ${ }^{82}$ that have been offset from each other for visual clarity. The wafers will be gold plated and bonded together into a monolithic feedhorn array before detectors are installed behind them.

By positioning the dewar window and the first lens near the ACT focus (Figure 4), we minimize the physical size of the optical elements; however, the increased FOV means that significantly larger optical elements are required than were used in MBAC. Because silicon has high thermal conductivity, a high index of refraction, and low loss at our wavelengths, it is the baseline for the ACTpol optics.

The optical requirements for illuminating a flat feedhorn array are also more stringent than for the free-space coupled bolometers used in MBAC. In particular, it is critical to have a telecentric focal plant to maintain symmetric beams and to preserve the polarization purity across the feedhorn array.

The increased throughput and telecentricity requirements of ACTPol drive the optical design to have more shaped surfaces than MBAC in order to achieve an optimized, diffraction-limited focal plane. By making both sides of the three ACTPol lenses curved and allowing the lenses to have small tilts and offsets, we are able to achieve high optical quality (Strehl ratios $>0.95$ ) across a large telecentric focal plane, as shown in Figure 4.

Two anti-reflection coatings are being considered for the ACTPol lenses. The first is replication of the CIRLEX coatings used in MBAC ${ }^{72}$ We calculate that the CIRLEX in MBAC causes $\sim 15 \%$ efficiency reduction per optics tube due to loss and reflections, so we are developing simulated dielectric anti-reflection coatings as an alternative. The simulated dielectrics are fabricated by removing much of the silicon material to a controlled depth from the surface of the lens at sub-wavelength scales, which reduces the effective index of refraction. Three approaches are being explored to achieve this: laser-machining, deep-reactive-ion-etching, and cutting with a silicon dicing saw. An optimized two-layer simulated dielectric coating is predicted to reduce the net loss to $<3 \%$ per optics tube. ${ }^{73}$

\subsection{Detector Arrays}

The ACTPol detectors couple to incoming radiation via monolithic corrugated feedhorn arrays coated with gold (Figure 5). Currently in development at NIST 8182 the feedhorn arrays will be assembled from individual $15 \mathrm{~cm}$ diameter silicon wafers with micro-machined circular apertures that correspond to individual corrugations. The silicon wafer platelets will then be stacked, bonded and gold-plated to form a close-packed feedhorn array. This approach preserves the advantages of corrugated feedhorns (low sidelobes, low cross-polarization, wide-band performance, etc.) while reducing the difficulty of building such a large array by use of traditional techniques (direct machining or electroforming individual metal feeds). While similar arrays can be made from metal platelets,

\footnotetext{
${ }^{\dagger \dagger}$ In a telecentric focal plane the chief ray originating from the center of the illuminated region on the primary mirror is perpendicular to the focal plane surface for all positions in the focal plane.
} 
considerations of weight, thermal mass, alignment, and differential thermal contraction with the detector arrays present difficulties that can be circumvented with the silicon platelet array concept.

A prototype $150 \mathrm{GHz}$ polarimeter is depicted in Figure 5 . The number of polarimeters that can fit into each array is limited to $\lesssim 650$ by the minimum practical spacing of each polarimeter $(\sim 5 \mathrm{~mm})$ and the maximum usable diameter of the micromachined silicon feedhorn array $(\sim 14 \mathrm{~cm})$. The polarimeters use superconducting transition-edge sensor (TES) bolometers to measure changes in radiation power. When the TES is appropriately voltage-biased, electrothermal feedback (ETF) maintains it at the transition temperature, $T_{c}$, under a wide range

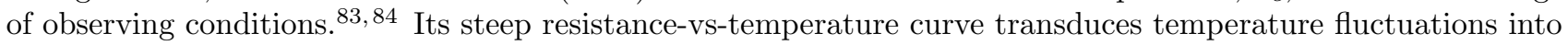
current fluctuations, which are read out by use of sensitive SQUID amplifiers in the time-division multiplexing architecture developed at NIST ${ }^{85}[86$ and implemented with room-temperature electronics provided by UBC. 87 The ETF speeds the response of the TES to temperature fluctuations compared to its natural thermal time constant. Each of the two TESes per polarimeter (one for each linear orthogonal polarization) is a molybdenumcopper bilayer with normal resistance $R_{n} \approx 6 \mathrm{~m} \Omega$, which absorbs power from a pair of microstrip transmission lines emanating from a planar orthomode transducer ${ }^{78}(\mathrm{OMT})$ and terminated in lossy gold meanders on the TES's thermally-isolated island. The island connects to the bulk, at temperature $T_{b}$, through four long legs composed of silicon nitride membrane topped with niobium wiring and sandwiched in silicon dioxide that have a total thermal conductivity $G$. Microstrip filters ${ }^{77}$ define the bandpasses. Gold resistors on the island are used as heaters that serve as responsivity calibration transfer standards and allow in situ measurements of the detector time constants. Each detector requires a shunt resistor $\left(R_{s} \approx 180 \mu \Omega\right)$ for voltage biasing and an inductor $(L \approx 1 \mu \mathrm{H})$ to lowpass-filter the current fluctuations.

Since April 2008, the Truce collaboration has designed, produced and tested three generations of fully functional polarimeters operating at $300 \mathrm{mK}]^{74}$ As reported in Bleem et al. ${ }^{77}$ we have measured the detector bandpass, the detector efficiency and the leakage between the orthogonal modes $(<3 \%)$. In Austermann et $a l . \frac{76]}{n}$ we report on the wafer-wide uniformity of $T_{c}( \pm 1 \%), R_{n}( \pm 10 \%), G( \pm 10 \%)$, and the detector time constants, $\tau( \pm 10 \%)$. We have modeled the TES by fitting to its complex impedance data and then predicted its noise performance to within $10-20 \% .75$ We are now building the first prototype polarimeter array with $\sim 100$ feedhorns and detectors 8288

\subsection{Sensitivity Forecast}

The baseline plan for the ACTPol receiver is to cool the detector arrays to a bath temperature of $T_{b} \approx 100 \mathrm{mK}$ rather than $300 \mathrm{mK}$ (as for MBAC). The lower temperature reduces the detector thermal noise below the photon noise. The latter is proportional to the loading; a detector sensitive to only one linear polarization mode receives half the loading of an unpolarized detector, which increases the payoff for reducing the thermal noise.

To achieve our science goals we target the map sensitivities indicated in $\$ 3$ for the ACT Wide II and Deep surveys. We introduce several conservative assumptions in calculating the detector sensitivities. Although we predict the yield on the installed detectors will be $90 \%$ or higher, for the calculations here we assume $70 \%$. We forecast realizing the same effective number of hours on the CMB per month that we achieved with MBAC in 2008. We make conservative (low) estimates of the optical efficiencies of the receiver based on measurements of components in hand, rather than using our predictions for improvements (in the detector efficiency, and possibly in the lenses); similarly we use the measured lower limit on the detector bandpass, which is $30 \%$ smaller than the design. These considerations lead to a goal for the noise equivalent power from thermal fluctuations in the detector of $\mathrm{NEP}_{G} \lesssim 2.0 \times 10^{-17} \mathrm{~W} / \sqrt{\mathrm{Hz}}$. Tests at NIST and Princeton $\left.{ }^{75} \mid 76\right]$ on prototype detectors indicate that their noise performance is straightforward to predict from their thermal conductance and is sufficiently low (but is not yet optimized for the expected ACTPol optical load or the target bath temperature).

We determine the target parameters for $T_{c}$ and $G$ consistent with the noise and detector time-constant goals. Our model for optimizing these parameters includes radiative loading and noise from the CMB, the atmosphere, the warm ACT mirrors, the dewar window, and cryogenic elements, as well as the detector noise, saturation power, $P_{\text {sat }}$, and bath temperature, $T_{b} . \sqrt[89]{ }$ Figure 6 illustrates how the NEP varies with $T_{c}$ for two sets of atmospheric loading conditions and two $P_{\text {sat }}$ values. The right panel shows the improvement in mapping speed from operating at $T_{b} \approx 100 \mathrm{mK}$ as compared to $\sim 300 \mathrm{mK}$. These calculations and the estimated number of 

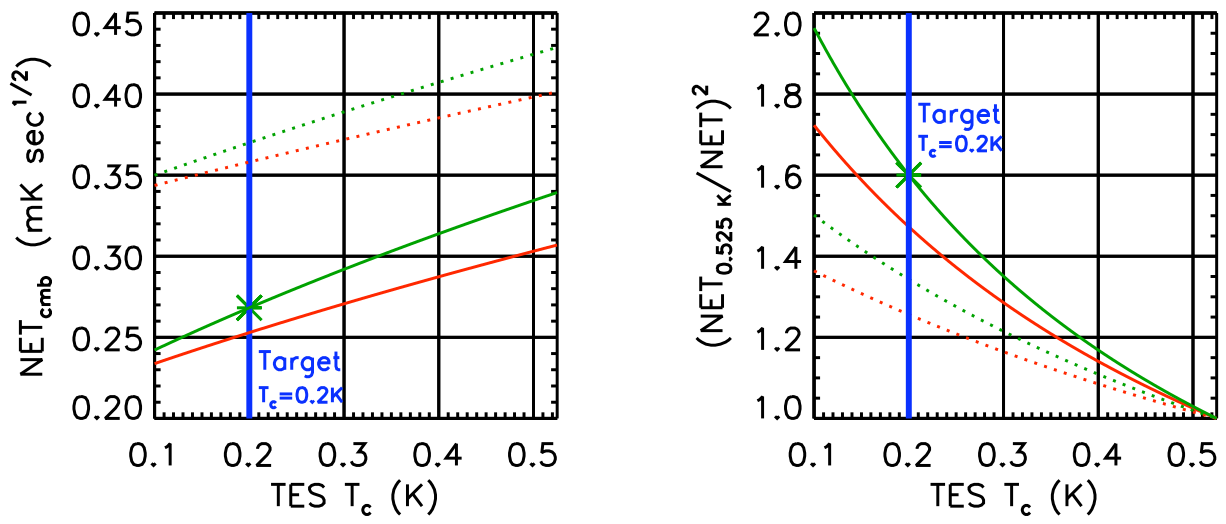

Figure 6. Improvement with $100 \mathrm{mK}$ vs $300 \mathrm{mK}$ cryogenics for a $150 \mathrm{GHz}$ detector. Left: the sensitivity of each TES bolometer to CMB temperature fluctuations under load as a function of the critical temperature $T_{c}$ of the TES. Right: the improvement in mapping speed as $T_{c}$ decreases; the abscissa gives the ratio of the weight after a fixed period observing with $T_{c}=0.525 \mathrm{~K}$ (as for $\sim 300 \mathrm{mK}$ cryogenics) to that from lower $T_{c}$. Here we have assumed $T_{c}=5 / 3 T_{b a t h}$ and optimized $G$ for the $P_{\text {sat }}$ target. In each plot, the green (red) curves are for $P_{\text {sat }}=10(7) \mathrm{pW}$, while the solid (dotted) curves are for $0.5(2.0) \mathrm{mm}$ of precipitable water vapor (PWV). The blue line indicates the targeted $T_{c}=0.2 \mathrm{~K}$, and the green asterisks indicate the sensitivity assumed for the error estimates in $\$ 2$ We will choose the $P_{\text {sat }}$ target after our initial prototyping runs of detectors with $T_{c}=0.2 \mathrm{~K}$; for now we conservatively estimate $P_{s a t} \approx 5 P_{\gamma}$, where $P_{\gamma}$ is the total photon load. The median PWV for the 2008 ACT observing season was $0.5 \mathrm{~mm}$.

working detectors lead to the target ACTPol instrument noise equivalent temperatures of $6 \mu \mathrm{K} \sqrt{\mathrm{s}}$ at $150 \mathrm{GHz}$ and $20 \mu \mathrm{K} \sqrt{\mathrm{s}}$ at $220 \mathrm{GHz}$.

\subsection{The ACTPol Cryostat}

The cryo-mechanical aspect of the ACTPol receiver will be similar to, but larger than, the existing MBAC receiver. The primary cooling for the receiver will be provided by two pulse tube refrigerators. Because the bandpasses for the ACTPol bolometers are defined on the detector wafers rather than by free-space filters, we need only cool the optical elements to $4 \mathrm{~K}$. Additional cooling stages are then required to cool only the detector arrays and to provide thermal isolation. The baseline plan for the cryostat includes three such stages. The first stage will be cooled to $\sim 0.8 \mathrm{~K}$ by a ${ }^{4} \mathrm{He}$ sorption refrigerator similar to the ones in MBAC. The second is a $300 \mathrm{mK}{ }^{3} \mathrm{He}$ sorption refrigerator, which will back single-stage adiabatic demagnetization refrigerators (ADRs) that cool the detector arrays to $100 \mathrm{mK}$. We predict $>30$ hour hold times for the ACTPol He fridges and > 50 hour hold times for the ADRs, which are more than two and three times longer than needed for observations.

\subsection{Improvements in the ACTPol Receiver Compared to the MBAC Receiver}

The ACTPol receiver is a considerable upgrade over MBAC. The most obvious improvement is that these detectors have polarization sensitivity that enables the new science described earlier. The colder operating temperature allows for lower NEPs over what is possible with $300 \mathrm{mK}$ cryogenics. The ACTPol field-of-view and optical throughput are both substantially larger than that of MBAC. We also are baselining two detector arrays, instead of one, at the critical science band of $150 \mathrm{GHz}$. In addition to having more detectors, this means there will be improved overlap of $150 \mathrm{GHz}$ and $220 \mathrm{GHz}$ observations on the sky.

Another substantial improvement is that we project the ACTPol detectors to have better noise performance than those in MBAC, which had excess in-band noise. The prototype ACTPol detectors exhibit in-band NEPs consistent with thermal fluctuation noise for $0.5<F_{\text {link }}<1,76$ whereas the MBAC detectors suffer from a median value of roughly $60 \%$ excess in-band noise compared to the model with $F_{\text {link }}=1$. 90

These combined improvements in detector NEP, optical throughput, number of detectors, and bath temperature result in the target ACTPol sensitivity being more than four times better than that of the current MBAC receiver for $150 \mathrm{GHz}$ temperature measurements, in addition to its polarization sensitivity. 

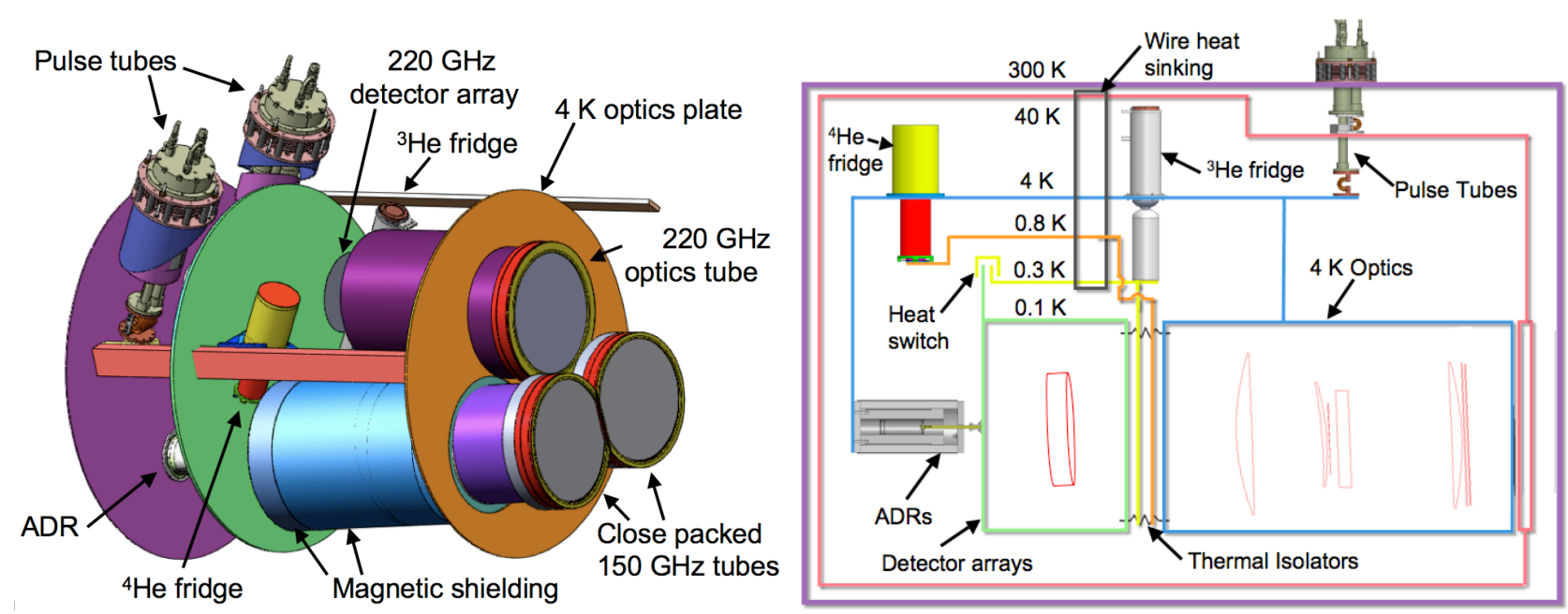

Figure 7. Left: Rendering of a preliminary ACTPol receiver design. The entrance of each optics tube is $\sim 30 \mathrm{~cm}$ diameter. Right: Schematic of cryogenic links in the receiver.

\section{CONCLUSION}

The development of the ACTPol receiver is underway, while CMB temperature observations with the existing MBAC receiver continue. ACTPol will observe both wide and deep fields, overlapping with a variety of observations accessible from the Atacama and enabling a wide range of science goals, including: measuring the gravitational lensing of the $\mathrm{CMB}$, constraining the sum of the neutrino masses and key parameters of inflation, and cross-correlating SZ and lensing measurements with other surveys. Target first light for ACTPol with three detector arrays is 2013.

\section{ACKNOWLEDGMENTS}

We thank members of the ACT and Truce collaborations for critical contributions to this project. ACT is supported primarily by the U.S. National Science Foundation through awards AST-0408698 for the ACT project, and PHY-0355328, AST-0707731 and PIRE-0507768. CMB detector development at NIST is supported by the NIST Innovations in Measurement Science program. Funding was also provided by Princeton University and the University of Pennsylvania. Niemack was supported by a National Research Council Postdoctoral Fellowship.

\section{REFERENCES}

[1] Fowler, J. W., Niemack, M. D., Dicker, S. R., Aboobaker, A. M., Ade, P. A. R., Battistelli, E. S., Devlin, M. J., Fisher, R. P., Halpern, M., Hargrave, P. C., Hincks, A. D., Kaul, M., Klein, J., Lau, J. M., Limon, M., Marriage, T. A., Mauskopf, P. D., Page, L., Staggs, S. T., Swetz, D. S., Switzer, E. R., Thornton, R. J., and Tucker, C. E., "Optical design of the Atacama Cosmology Telescope and the Millimeter Bolometric Array Camera," Appl. Opt. 46(17), 3444-3454 (2007).

[2] The ACT Collaboration, Fowler, J. W., Acquaviva, V., Ade, P. A. R., Aguirre, P., Amiri, M., Appel, J. W., Barrientos, L. F., Battistelli, E. S., Bond, J. R., Brown, B., Burger, B., Chervenak, J., Das, S., Devlin, M. J., Dicker, S. R., Doriese, W. B., Dunkley, J., Dünner, R., Essinger-Hileman, T., Fisher, R. P., Hajian, A., Halpern, M., Hasselfield, M., Hernández-Monteagudo, C., Hilton, G. C., Hilton, M., Hincks, A. D., Hlozek, R., Huffenberger, K. M., Hughes, D. H., Hughes, J. P., Infante, L., Irwin, K. D., Jimenez, R., Juin, J. B., Kaul, M., Klein, J., Kosowsky, A., Lau, J. M., Limon, M., Lin, Y., Lupton, R. H., Marriage, T. A., Marsden, D., Martocci, K., Mauskopf, P., Menanteau, F., Moodley, K., Moseley, H., Netterfield, C. B., Niemack, M. D., Nolta, M. R., Page, L. A., Parker, L., Partridge, B., Quintana, H., Reid, B., Sehgal, N., Sievers, J., Spergel, D. N., Staggs, S. T., Swetz, D. S., Switzer, E. R., Thornton, R., Trac, H., Tucker, C., Verde, L., Warne, R., Wilson, G., Wollack, E., and Zhao, Y., "The Atacama Cosmology Telescope: A 
Measurement of the $600<l<8000$ Cosmic Microwave Background Power Spectrum at $148 \mathrm{GHz}$," ArXiv e-prints , 1001.2934 (Jan. 2010).

[3] Hincks, A. D., Acquaviva, V., Ade, P., Aguirre, P., Amiri, M., Appel, J. W., Barrientos, L. F., Battistelli, E. S., Bond, J. R., Brown, B., Burger, B., Chervenak, J., Das, S., Devlin, M. J., Dicker, S., Doriese, W. B., Dunkley, J., Dünner, R., Essinger-Hileman, T., Fisher, R. P., Fowler, J. W., Hajian, A., Halpern, M., Hasselfield, M., Hernández-Monteagudo, C., Hilton, G. C., Hilton, M., Hlozek, R., Huffenberger, K., Hughes, D., Hughes, J. P., Infante, L., Irwin, K. D., Jimenez, R., Juin, J. B., Kaul, M., Klein, J., Kosowsky, A., Lau, J. M., Limon, M., Lin, Y., Lupton, R. H., Marriage, T., Marsden, D., Martocci, K., Mauskopf, P., Menanteau, F., Moodley, K., Moseley, H., Netterfield, C. B., Niemack, M. D., Nolta, M. R., Page, L. A., Parker, L., Partridge, B., Quintana, H., Reid, B., Sehgal, N., Sievers, J., Spergel, D. N., Staggs, S. T., Stryzak, O., Swetz, D., Switzer, E., Thornton, R., Trac, H., Tucker, C., Verde, L., Warne, R., Wilson, G., Wollack, E., and Zhao, Y., "The Atacama Cosmology Telescope (ACT): Beam Profiles and First SZ Cluster Maps," ArXiv e-prints , 0907.0461 (July 2009).

[4] Brown, M. L., Ade, P., Bock, J., Bowden, M., Cahill, G., Castro, P. G., Church, S., Culverhouse, T., Friedman, R. B., Ganga, K., Gear, W. K., Gupta, S., Hinderks, J., Kovac, J., Lange, A. E., Leitch, E., Melhuish, S. J., Memari, Y., Murphy, J. A., Orlando, A., O'Sullivan, C., Piccirillo, L., Pryke, C., Rajguru, N., Rusholme, B., Schwarz, R., Taylor, A. N., Thompson, K. L., Turner, A. H., Wu, E. Y. S., Zemcov, M., and The QUa D collaboration, "Improved Measurements of the Temperature and Polarization of the Cosmic Microwave Background from QUaD," Astrophys. J. 705, 978-999 (Nov. 2009).

[5] Chiang, H. C., Ade, P. A. R., Barkats, D., Battle, J. O., Bierman, E. M., Bock, J. J., Dowell, C. D., Duband, L., Hivon, E. F., Holzapfel, W. L., Hristov, V. V., Jones, W. C., Keating, B. G., Kovac, J. M., Kuo, C. L., Lange, A. E., Leitch, E. M., Mason, P. V., Matsumura, T., Nguyen, H. T., Ponthieu, N., Pryke, C., Richter, S., Rocha, G., Sheehy, C., Takahashi, Y. D., Tolan, J. E., and Yoon, K. W., "Measurement of Cosmic Microwave Background Polarization Power Spectra from Two Years of BICEP Data," Astrophys. J. 711, 1123-1140 (Mar. 2010).

[6] Montroy, T. E., Ade, P. A. R., Bock, J. J., Bond, J. R., Borrill, J., Boscaleri, A., Cabella, P., Contaldi, C. R., Crill, B. P., de Bernardis, P., De Gasperis, G., de Oliveira-Costa, A., De Troia, G., di Stefano, G., Hivon, E., Jaffe, A. H., Kisner, T. S., Jones, W. C., Lange, A. E., Masi, S., Mauskopf, P. D., MacTavish, C. J., Melchiorri, A., Natoli, P., Netterfield, C. B., Pascale, E., Piacentini, F., Pogosyan, D., Polenta, G., Prunet, S., Ricciardi, S., Romeo, G., Ruhl, J. E., Santini, P., Tegmark, M., Veneziani, M., and Vittorio, N., "A Measurement of the CMB EE Spectrum from the 2003 Flight of BOOMERANG," Astrophys. J. 647, 813-822 (Aug. 2006).

[7] Bischoff, C., Hyatt, L., McMahon, J. J., Nixon, G. W., Samtleben, D., Smith, K. M., Vanderlinde, K., Barkats, D., Farese, P., Gaier, T., Gundersen, J. O., Hedman, M. M., Staggs, S. T., and Winstein, B., "New Measurements of Fine-Scale CMB Polarization Power Spectra from CAPMAP at Both 40 and $90 \mathrm{GHz}$," Astrophys. J. 684, 771-789 (Sept. 2008).

[8] Sievers, J. L., Achermann, C., Bond, J. R., Bronfman, L., Bustos, R., Contaldi, C. R., Dickinson, C., Ferreira, P. G., Jones, M. E., Lewis, A. M., Mason, B. S., May, J., Myers, S. T., Oyarce, N., Padin, S., Pearson, T. J., Pospieszalski, M., Readhead, A. C. S., Reeves, R., Taylor, A. C., and Torres, S., "Implications of the Cosmic Background Imager Polarization Data," Astrophys. J. 660, 976-987 (May 2007).

[9] Kovac, J. M., Leitch, E. M., Pryke, C., Carlstrom, J. E., Halverson, N. W., and Holzapfel, W. L., "Detection of polarization in the cosmic microwave background using DASI," Nature 420, 772-787 (Dec. 2002).

[10] Nolta, M. R., Dunkley, J., Hill, R. S., Hinshaw, G., Komatsu, E., Larson, D., Page, L., Spergel, D. N., Bennett, C. L., Gold, B., Jarosik, N., Odegard, N., Weiland, J. L., Wollack, E., Halpern, M., Kogut, A., Limon, M., Meyer, S. S., Tucker, G. S., and Wright, E. L., "Five-Year Wilkinson Microwave Anisotropy Probe Observations: Angular Power Spectra," Astrophys. J. Supp. 180, 296-305 (Feb. 2009).

[11] Knox, L., "Determination of inflationary observables by cosmic microwave background anisotropy experiments," Phys. Rev. D 52, 4307-4318 (Oct. 1995).

[12] Guth, A. H., "Inflationary universe: A possible solution to the horizon and flatness problems," Phys. Rev. D 23, 347 (1981).

[13] Linde, A. D., "A new inflationary universe scenario: A possible solution of the horizon, flatness, homogeneity, isotropy and primordial monopole problems," Phys. Lett. B108, 389-393 (1982). 
[14] Albrecht, A. and Steinhardt, P. J., "Cosmology for grand unified theories with radiatively induced symmetry breaking," PRL 48, 1220-1223 (Apr. 1982).

[15] Sato, K., "First-order phase transition of a vacuum and the expansion of the Universe," Monthly Not. of Royal Astron. Soc. 195, 467-479 (May 1981).

[16] Mukhanov, V. F. and Chibisov, G. V., "Quantum fluctuations and a nonsingular universe," JETP Letters 33, 532 (May 1981).

[17] Hawking, S. W., "The development of irregularities in a single bubble inflationary universe," Phys. Lett. B115, 295 (1982).

[18] Guth, A. H. and Pi, S. Y., "Fluctuations in the new inflationary universe," Phys. Rev. Lett. 49, 1110-1113 (1982).

[19] Starobinsky, A. A., "Dynamics of phase transition in the new inflationary universe scenario and generation of perturbations," Phys. Lett. B117, 175-178 (1982).

[20] Bardeen, J. M., Steinhardt, P. J., and Turner, M. S., "Spontaneous creation of almost scale-free density perturbations in an inflationary universe," Phys. Rev. D 28, 679 (1983).

[21] Kosowsky, A. and Turner, M. S., "CBR anisotropy and the running of the scalar spectral index," Phys. Rev. D 52, 1739- (Aug. 1995).

[22] Komatsu, E., "Hunting for Primordial Non-Gaussianity in the Cosmic Microwave Background," ArXiv e-prints , 1003.6097 (Mar. 2010).

[23] Fraisse, A. A., Ringeval, C., Spergel, D. N., and Bouchet, F. R., "Small-angle CMB temperature anisotropies induced by cosmic strings," Phys. Rev. D 78, 043535 (Aug. 2008).

[24] Ichikawa, K., Sekiguchi, T., and Takahashi, T., "Primordial helium abundance from CMB: A constraint from recent observations and a forecast," Phys. Rev. D 78, 043509 (Aug. 2008).

[25] Iocco, F., Mangano, G., Miele, G., Pisanti, O., and Serpico, P. D., "Primordial nucleosynthesis: From precision cosmology to fundamental physics," Phys. Rep. 472, 1-76 (Mar. 2009).

[26] Fukugita, M. and Kawasaki, M., "Primordial Helium Abundance: A Reanalysis of the Izotov-Thuan Spectroscopic Sample," Astrophys. J. 646, 691-695 (Aug. 2006).

[27] Dunkley, J., Komatsu, E., Nolta, M. R., Spergel, D. N., Larson, D., Hinshaw, G., Page, L., Bennett, C. L., Gold, B., Jarosik, N., Weiland, J. L., Halpern, M., Hill, R. S., Kogut, A., Limon, M., Meyer, S. S., Tucker, G. S., Wollack, E., and Wright, E. L., "Five-Year Wilkinson Microwave Anisotropy Probe (WMAP) Observations: Likelihoods and Parameters from the WMAP data," Astrophys. J. Supp. 180, 306-376 (Feb. 2009).

[28] Adamson, P., Andreopoulos, C., Arms, K. E., Armstrong, R., Auty, D. J., Ayres, D. S., Baller, B., Barnes, Jr., P. D., Barr, G., Barrett, W. L., Becker, B. R., Belias, A., Bernstein, R. H., Bhattacharya, D., Bishai, M., Blake, A., Bock, G. J., Boehm, J., Boehnlein, D. J., Bogert, D., Bower, C., Buckley-Geer, E., Cavanaugh, S., Chapman, J. D., Cherdack, D., Childress, S., Choudhary, B. C., Cobb, J. H., Coleman, S. J., Culling, A. J., de Jong, J. K., Dierckxsens, M., Diwan, M. V., Dorman, M., Dytman, S. A., Escobar, C. O., Evans, J. J., Falk Harris, E., Feldman, G. J., Frohne, M. V., Gallagher, H. R., Godley, A., Goodman, M. C., Gouffon, P., Gran, R., Grashorn, E. W., Grossman, N., Grzelak, K., Habig, A., Harris, D., Harris, P. G., Hartnell, J., Hatcher, R., Heller, K., Himmel, A., Holin, A., Hylen, J., Irwin, G. M., Ishitsuka, M., Jaffe, D. E., James, C., Jensen, D., Kafka, T., Kasahara, S. M. S., Kim, J. J., Kim, M. S., Koizumi, G., Kopp, S., Kordosky, M., Koskinen, D. J., Kotelnikov, S. K., Kreymer, A., Kumaratunga, S., Lang, K., Ling, J., Litchfield, P. J., Litchfield, R. P., Loiacono, L., Lucas, P., Ma, J., Mann, W. A., Marchionni, A., Marshak, M. L., Marshall, J. S., Mayer, N., McGowan, A. M., Meier, J. R., Merzon, G. I., Messier, M. D., Metelko, C. J., Michael, D. G., Miller, J. L., Miller, W. H., Mishra, S. R., Moore, C. D., Morfín, J., Mualem, L., Mufson, S., Murgia, S., Musser, J., Naples, D., Nelson, J. K., Newman, H. B., Nichol, R. J., Nicholls, T. C., Ochoa-Ricoux, J. P., Oliver, W. P., Ospanov, R., Paley, J., Paolone, V., Para, A., Patzak, T., Pavlović, Ž., Pawloski, G., Pearce, G. F., Peck, C. W., Peterson, E. A., Petyt, D. A., Pittam, R., Plunkett, R. K., Rahaman, A., Rameika, R. A., Raufer, T. M., Rebel, B., Reichenbacher, J., Rodrigues, P. A., Rosenfeld, C., Rubin, H. A., Ruddick, K., Ryabov, V. A., Sanchez, M. C., Saoulidou, N., Schneps, J., Schreiner, P., Seun, S.-M., Shanahan, P., Smart, W., Smith, C., Sousa, A., Speakman, B., Stamoulis, P., Strait, M., Symes, P., Tagg, N., Talaga, R. L., Tavera, M. A., Thomas, J., Thompson, J., Thomson, M. A., Thron, J. L., Tinti, 
G., Trostin, I., Tsarev, V. A., Tzanakos, G., Urheim, J., Vahle, P., Viren, B., Ward, C. P., Ward, D. R., Watabe, M., Weber, A., Webb, R. C., Wehmann, A., West, N., White, C., Wojcicki, S. G., Wright, D. M., Yang, T., Zois, M., Zhang, K., and Zwaska, R., "Measurement of Neutrino Oscillations with the MINOS Detectors in the NuMI Beam," Physical Review Letters 101, 131802 (Sept. 2008).

[29] Jimenez, R., Kitching, T., Peña-Garay, C., and Verde, L., "Can we measure the neutrino mass hierarchy in the sky?," Journal of Cosmology and Astro-Particle Physics 5, 35-+ (May 2010).

[30] Bashinsky, S. and Seljak, U., "Signatures of relativistic neutrinos in CMB anisotropy and matter clustering," Phys. Rev. D 69, 083002 (Apr. 2004).

[31] Komatsu, E., Smith, K. M., Dunkley, J., Bennett, C. L., Gold, B., Hinshaw, G., Jarosik, N., Larson, D., Nolta, M. R., Page, L., Spergel, D. N., Halpern, M., Hill, R. S., Kogut, A., Limon, M., Meyer, S. S., Odegard, N., Tucker, G. S., Weiland, J. L., Wollack, E., and Wright, E. L., "Seven-Year Wilkinson Microwave Anisotropy Probe (WMAP) Observations: Cosmological Interpretation," ArXiv e-prints (Jan. 2010).

[32] Zaldarriaga, M. and Seljak, U., "Gravitational Lensing Effect on Cosmic Microwave Background Polarization," Phys. Rev. D58, 023003 (1998).

[33] de Putter, R., Zahn, O., and Linder, E. V., "CMB lensing constraints on neutrinos and dark energy," Phys. Rev. D 79, 065033 (Mar. 2009).

[34] Smith, K. M., Cooray, A., Das, S., Doré, O., Hanson, D., Hirata, C., Kaplinghat, M., Keating, B., LoVerde, M., Miller, N., Rocha, G., Shimon, M., and Zahn, O., "CMBPol Mission Concept Study: Gravitational Lensing," ArXiv e-prints, 0811.3916 (Nov. 2008).

[35] Stompor, R. and Efstathiou, G., "Gravitational lensing of cosmic microwave background anisotropies and cosmological parameter estimation," Monthly Not. of Royal Astron. Soc. 302, 735-747 (Feb. 1999).

[36] Vallinotto, A., Das, S., Spergel, D. N., and Viel, M., "Lenses in the Forest: Cross Correlation of the Lyman- $\alpha$ Flux with Cosmic Microwave Background Lensing," Physical Review Letters 103, 091304 (Aug. 2009).

[37] Percival, W. J., Reid, B. A., Eisenstein, D. J., Bahcall, N. A., Budavari, T., Frieman, J. A., Fukugita, M., Gunn, J. E., Ivezić, Ž., Knapp, G. R., Kron, R. G., Loveday, J., Lupton, R. H., McKay, T. A., Meiksin, A., Nichol, R. C., Pope, A. C., Schlegel, D. J., Schneider, D. P., Spergel, D. N., Stoughton, C., Strauss, M. A., Szalay, A. S., Tegmark, M., Vogeley, M. S., Weinberg, D. H., York, D. G., and Zehavi, I., "Baryon acoustic oscillations in the Sloan Digital Sky Survey Data Release 7 galaxy sample," Monthly Not. of Royal Astron. Soc. 401, 2148-2168 (Feb. 2010).

[38] Reid, B. A., Percival, W. J., Eisenstein, D. J., Verde, L., Spergel, D. N., Skibba, R. A., Bahcall, N. A., Budavari, T., Frieman, J. A., Fukugita, M., Gott, J. R., Gunn, J. E., Ivezić, Ž., Knapp, G. R., Kron, R. G., Lupton, R. H., McKay, T. A., Meiksin, A., Nichol, R. C., Pope, A. C., Schlegel, D. J., Schneider, D. P., Stoughton, C., Strauss, M. A., Szalay, A. S., Tegmark, M., Vogeley, M. S., Weinberg, D. H., York, D. G., and Zehavi, I., "Cosmological constraints from the clustering of the Sloan Digital Sky Survey DR7 luminous red galaxies," Monthly Not. of Royal Astron. Soc. 404, 60-85 (May 2010).

[39] Acquaviva, V., Hajian, A., Spergel, D. N., and Das, S., "Next generation redshift surveys and the origin of cosmic acceleration," PRD 78, 043514 (aug 2009).

[40] Sunyaev, R. A. and Zeldovich, I. B., "Microwave background radiation as a probe of the contemporary structure and history of the universe," Annual Rev. of Astron. and Astrophys. 18, 537-560 (1980).

[41] Staniszewski, Z., Ade, P. A. R., Aird, K. A., Benson, B. A., Bleem, L. E., Carlstrom, J. E., Chang, C. L., Cho, H., Crawford, T. M., Crites, A. T., de Haan, T., Dobbs, M. A., Halverson, N. W., Holder, G. P., Holzapfel, W. L., Hrubes, J. D., Joy, M., Keisler, R., Lanting, T. M., Lee, A. T., Leitch, E. M., Loehr, A., Lueker, M., McMahon, J. J., Mehl, J., Meyer, S. S., Mohr, J. J., Montroy, T. E., Ngeow, C., Padin, S., Plagge, T., Pryke, C., Reichardt, C. L., Ruhl, J. E., Schaffer, K. K., Shaw, L., Shirokoff, E., Spieler, H. G., Stalder, B., Stark, A. A., Vanderlinde, K., Vieira, J. D., Zahn, O., and Zenteno, A., "Galaxy Clusters Discovered with a Sunyaev-Zel'dovich Effect Survey," Astrophys. J. 701, 32-41 (Aug. 2009).

[42] Sehgal, N., Bode, P., Das, S., Hernandez-Monteagudo, C., Huffenberger, K., Lin, Y., Ostriker, J. P., and Trac, H., "Simulations of the Microwave Sky," Astrophys. J. 709, 920-936 (Feb. 2010).

[43] Tinker, J., Kravtsov, A. V., Klypin, A., Abazajian, K., Warren, M., Yepes, G., Gottlöber, S., and Holz, D. E., "Toward a Halo Mass Function for Precision Cosmology: The Limits of Universality," Astrophys. J. 688, 709-728 (Dec. 2008). 
[44] Majumdar, S. and Mohr, J. J., "Self-Calibration in Cluster Studies of Dark Energy: Combining the Cluster Redshift Distribution, the Power Spectrum, and Mass Measurements," Astrophys. J. 613, 41-50 (Sept. 2004).

[45] Vanderlinde, K., Crawford, T. M., de Haan, T., Dudley, J. P., Shaw, L., Ade, P. A. R., Aird, K. A., Benson, B. A., Bleem, L. E., Brodwin, M., Carlstrom, J. E., Chang, C. L., Crites, A. T., Desai, S., Dobbs, M. A., Foley, R. J., George, E. M., Gladders, M. D., Hall, N. R., Halverson, N. W., High, F. W., Holder, G. P., Holzapfel, W. L., Hrubes, J. D., Joy, M., Keisler, R., Knox, L., Lee, A. T., Leitch, E. M., Loehr, A., Lueker, M., Marrone, D. P., McMahon, J. J., Mehl, J., Meyer, S. S., Mohr, J. J., Montroy, T. E., Ngeow, C., Padin, S., Plagge, T., Pryke, C., Reichardt, C. L., Rest, A., Ruel, J., Ruhl, J. E., Schaffer, K. K., Shirokoff, E., Song, J., Spieler, H. G., Stalder, B., Staniszewski, Z., Stark, A. A., Stubbs, C. W., van Engelen, A., Vieira, J. D., Williamson, R., Yang, Y., Zahn, O., and Zenteno, A., "Galaxy Clusters Selected with the Sunyaev-Zel'dovich Effect from 2008 South Pole Telescope Observations," ArXiv e-prints, 1003.0003 (Mar. 2010).

[46] Khedekar, S., Majumdar, S., and Das, S., "Precision cosmology with a combination of wide and deep Sunyaev-Zeldovich cluster surveys," ArXiv e-prints (May 2010).

[47] Ho, S., Dedeo, S., and Spergel, D., "Finding the Missing Baryons Using CMB as a Backlight," ArXiv e-prints, 0903.2845 (Mar. 2009).

[48] Chatterjee, S., di Matteo, T., Kosowsky, A., and Pelupessy, I., "Simulations of the Sunyaev-Zel'dovich effect from quasars," Monthly Not. of Royal Astron. Soc. 390, 535-544 (Oct. 2008).

[49] Kamionkowski, M. and Loeb, A., "Getting around cosmic variance," Phys. Rev. D 56, 4511-4513 (Oct. 1997).

[50] Menanteau, F., Hughes, J. P., Barrientos, L. F., Deshpande, A. J., Hilton, M., Infante, L., Jimenez, R., Kosowsky, A., Moodley, K., Spergel, D., and Verde, L., "Southern Cosmology Survey II: Massive OpticallySelected Clusters from 70 square degrees of the SZE Common Survey Area," ArXiv e-prints (Feb. 2010).

[51] Richards, G. T., Myers, A. D., Gray, A. G., Riegel, R. N., Nichol, R. C., Brunner, R. J., Szalay, A. S., Schneider, D. P., and Anderson, S. F., "Efficient Photometric Selection of Quasars from the Sloan Digital Sky Survey. II. 1,000,000 Quasars from Data Release 6," Astrophys. J. Supp. 180, 67-83 (Jan. 2009).

[52] Koester, B. P., McKay, T. A., Annis, J., Wechsler, R. H., Evrard, A., Bleem, L., Becker, M., Johnston, D., Sheldon, E., Nichol, R., Miller, C., Scranton, R., Bahcall, N., Barentine, J., Brewington, H., Brinkmann, J., Harvanek, M., Kleinman, S., Krzesinski, J., Long, D., Nitta, A., Schneider, D. P., Sneddin, S., Voges, W., and York, D., "A MaxBCG Catalog of 13,823 Galaxy Clusters from the Sloan Digital Sky Survey," Astrophys. J. 660, 239-255 (May 2007).

[53] Condon, J. J., Cotton, W. D., Greisen, E. W., Yin, Q. F., Perley, R. A., Taylor, G. B., and Broderick, J. J., "The NRAO VLA Sky Survey," Astron. J. 115, 1693-1716 (May 1998).

[54] Vieira, J. D., Crawford, T. M., Switzer, E. R., Ade, P. A. R., Aird, K. A., Ashby, M. L. N., Benson, B. A., Bleem, L. E., Brodwin, M., Carlstrom, J. E., Chang, C. L., Cho, H., Crites, A. T., de Haan, T., Dobbs, M. A., Everett, W., George, E. M., Gladders, M., Hall, N. R., Halverson, N. W., High, F. W., Holder, G. P., Holzapfel, W. L., Hrubes, J. D., Joy, M., Keisler, R., Knox, L., Lee, A. T., Leitch, E. M., Lueker, M., Marrone, D. P., McIntyre, V., McMahon, J. J., Mehl, J., Meyer, S. S., Mohr, J. J., Montroy, T. E., Padin, S., Plagge, T., Pryke, C., Reichardt, C. L., Ruhl, J. E., Schaffer, K. K., Shaw, L., Shirokoff, E., Spieler, H. G., Stalder, B., Staniszewski, Z., Stark, A. A., Vanderlinde, K., Walsh, W., Williamson, R., Yang, Y., Zahn, O., and Zenteno, A., "Extragalactic millimeter-wave sources in South Pole Telescope survey data: source counts, catalog, and statistics for an 87 square-degree field," ArXiv e-prints, 0912.2338 (Dec. 2009).

[55] Murphy, T., Sadler, E. M., Ekers, R. D., Massardi, M., Hancock, P. J., Mahony, E., Ricci, R., Burke-Spolaor, S., Calabretta, M., Chhetri, R., de Zotti, G., Edwards, P. G., Ekers, J. A., Jackson, C. A., Kesteven, M. J., Lindley, E., Newton-McGee, K., Phillips, C., Roberts, P., Sault, R. J., Staveley-Smith, L., Subrahmanyan, R., Walker, M. A., and Wilson, W. E., "The Australia Telescope 20 GHz Survey: the source catalogue," Monthly Not. of Royal Astron. Soc. 402, 2403-2423 (Mar. 2010).

[56] Seiffert, M., Borys, C., Scott, D., and Halpern, M., "An upper limit to polarized submillimetre emission in Arp 220," Monthly Not. of Royal Astron. Soc. 374, 409-414 (Jan. 2007). 
[57] Tucci, M., Martínez-González, E., Vielva, P., and Delabrouille, J., "Limits on the detectability of the CMB B-mode polarization imposed by foregrounds," Monthly Not. of Royal Astron. Soc. 360, 935-949 (July 2005).

[58] Dunkley, J., Amblard, A., Baccigalupi, C., Betoule, M., Chuss, D., Cooray, A., Delabrouille, J., Dickinson, C., Dobler, G., Dotson, J., Eriksen, H. K., Finkbeiner, D., Fixsen, D., Fosalba, P., Fraisse, A., Hirata, C., Kogut, A., Kristiansen, J., Lawrence, C., Magalha Es, A. M., Miville-Deschenes, M. A., Meyer, S., Miller, A., Naess, S. K., Page, L., Peiris, H. V., Phillips, N., Pierpaoli, E., Rocha, G., Vaillancourt, J. E., and Verde, L., "Prospects for polarized foreground removal," in [American Institute of Physics Conference Series], S. Dodelson, D. Baumann, A. Cooray, J. Dunkley, A. Fraisse, M. G. Jackson, A. Kogut, L. Krauss, M. Zaldarriaga, \& K. Smith, ed., American Institute of Physics Conference Series 1141, 222-264 (June 2009).

[59] Hinshaw, G., Weiland, J. L., Hill, R. S., Odegard, N., Larson, D., Bennett, C. L., Dunkley, J., Gold, B., Greason, M. R., Jarosik, N., Komatsu, E., Nolta, M. R., Page, L., Spergel, D. N., Wollack, E., Halpern, M., Kogut, A., Limon, M., Meyer, S. S., Tucker, G. S., and Wright, E. L., "Five-Year Wilkinson Microwave Anisotropy Probe Observations: Data Processing, Sky Maps, and Basic Results," Astrophys. J. Supp. 180, 225-245 (Feb. 2009).

[60] Battye, R. A., Browne, I. W. A., Peel, M. W., Jackson, N. J., and Dickinson, C., "Statistical properties of polarized radio sources at high frequency and their impact on CMB polarization measurements," ArXiv e-prints (Mar. 2010).

[61] Hill, R. S., Weiland, J. L., Odegard, N., Wollack, E., Hinshaw, G., Larson, D., Bennett, C. L., Halpern, M., Page, L., Dunkley, J., Gold, B., Jarosik, N., Kogut, A., Limon, M., Nolta, M. R., Spergel, D. N., Tucker, G. S., and Wright, E. L., "Five-Year Wilkinson Microwave Anisotropy Probe Observations: Beam Maps and Window Functions," Astrophys. J. Supp. 180, 246-264 (Feb. 2009).

[62] Weiland, J. L., Odegard, N., Hill, R. S., Wollack, E., Hinshaw, G., Greason, M. R., Jarosik, N., Page, L., Bennett, C. L., Dunkley, J., Gold, B., Halpern, M., Kogut, A., Komatsu, E., Larson, D., Limon, M., Meyer, S. S., Nolta, M. R., Smith, K. M., Spergel, D. N., Tucker, G. S., and Wright, E. L., "Seven-Year Wilkinson Microwave Anisotropy Probe (WMAP) Observations: Planets and Celestial Calibration Sources," ArXiv e-prints (Jan. 2010).

[63] Swetz, D. S., Ade, P. A. R., Allen, C., Amiri, M., Appel, J. W., Battistelli, E. S., Burger, B., Chervenak, J. A., Dahlen, A. J., Das, S., Denny, S., Devlin, M. J., Dicker, S. R., Doriese, W. B., Dünner, R., EssingerHileman, T., Fisher, R. P., Fowler, J. W., Gao, X., Hajian, A., Halpern, M., Hargrave, P. C., Hasselfield, M., Hilton, G. C., Hincks, A. D., Irwin, K. D., Jarosik, N., Kaul, M., Klein, J., Knotek, S., Lau, J. M., Limon, M., Lupton, R. H., Marriage, T. A., Martocci, K. L., Mauskopf, P., Moseley, S. H., Netterfield, C. B., Niemack, M. D., Nolta, M. R., Page, L., Parker, L. P., Reid, B. A., Reintsema, C. D., Sederberg, A. J., Sehgal, N., Sievers, J. L., Spergel, D. N., Staggs, S. T., Stryzak, O. R., Switzer, E. R., Thornton, R. J., Tucker, C., Wollack, E. J., and Zhao, Y., "Instrument design and characterization of the Millimeter Bolometer Array Camera on the Atacama Cosmology Telescope," in [Proc. SPIE], Duncan, W. D., Holland, W. S., Withington, S., and Zmuidzinas, J., eds., Millimeter and Submillimeter Detectors and Instrumentation for Astronomy IV 7020, 702008, SPIE (2008).

[64] Thornton, R. J., Ade, P. A. R., Allen, C., Amiri, M., Appel, J. W., Battistelli, E. S., Burger, B., Chervenak, J. A., Devlin, M. J., Dicker, S. R., Doriese, W. B., Essinger-Hileman, T., Fisher, R. P., Fowler, J. W., Halpern, M., Hargrave, P. C., Hasselfield, M., Hilton, G. C., Hincks, A. D., Irwin, K. D., Jarosik, N., Kaul, M., Klein, J., Lau, J. M., Limon, M., Marriage, T. A., Martocci, K. L., Mauskopf, P., Moseley, S. H., Niemack, M. D., Page, L., Parker, L. P., Reidel, J., Reintsema, C. D., Staggs, S. T., Stryzak, O. R., Swetz, D. S., Switzer, E. R., Tucker, C., Wollack, E. J., and Zhao, Y., "Opto-mechanical design and performance of a compact three-frequency camera for the Millimeter Bolometer Array Camera on the Atacama Cosmology Telescope," in [Proc. SPIE], Duncan, W. D., Holland, W. S., Withington, S., and Zmuidzinas, J., eds., Millimeter and Submillimeter Detectors and Instrumentation for Astronomy IV 7020, 70201R, SPIE (2008).

[65] Niemack, M. D., Zhao, Y., Wollack, E., Thornton, R., Switzer, E. R., Swetz, D. S., Staggs, S. T., Page, L., Stryzak, O., Moseley, H., Marriage, T. A., Limon, M., Lau, J. M., Klein, J., Kaul, M., Jarosik, N., Irwin, K. D., Hincks, A. D., Hilton, G. C., Halpern, M., Fowler, J. W., Fisher, R. P., Dünner, R., Doriese, 
W. B., Dicker, S. R., Devlin, M. J., Chervenak, J., Burger, B., Battistelli, E. S., Appel, J., Amiri, M., Allen, C., and Aboobaker, A. M., "A kilopixel array of TES bolometers for ACT: Development, testing, and first light," J. Low Temp. Phys. 151(3-4), 690-696 (2008).

[66] Zhao, Y., Allen, C., Amiri, M., Appel, J. W., Battistelli, E. S., Burger, B., Chervenak, J. A., Dahlen, A. J., Denny, S., Devlin, M. J., Dicker, S. R., Doriese, W. B., Dünner, R., Essinger-Hileman, T., Fisher, R. P., Fowler, J. W., Halpern, M., Hilton, G. C., Hincks, A. D., Irwin, K. D., Jarosik, N., Klein, J., Lau, J. M., Marriage, T. A., Martocci, K. L., Moseley, S. H., Niemack, M. D., Page, L., Parker, L. P., Sederberg, A., Staggs, S. T., Stryzak, O. R., Swetz, D. S., Switzer, E. R., Thornton, R. J., and Wollack, E. J., "Characterization of transition edge sensors for the Millimeter Bolometer Array Camera on the Atacama Cosmology Telescope," in [Proc. SPIE], Duncan, W. D., Holland, W. S., Withington, S., and Zmuidzinas, J., eds., Millimeter and Submillimeter Detectors and Instrumentation for Astronomy IV 7020, 70200O, SPIE (2008).

[67] Switzer, E. R., Allen, C., Amiri, M., Appel, J. W., Battistelli, E. S., Burger, B., Chervenak, J. A., Dahlen, A. J., Das, S., Devlin, M. J., Dicker, S. R., Doriese, W. B., Dünner, R., Essinger-Hileman, T., Gao, X., Halpern, M., Hasselfield, M., Hilton, G. C., Hincks, A. D., Irwin, K. D., Knotek, S., Fisher, R. P., Fowler, J. W., Jarosik, N., Kaul, M., Klein, J., Lau, J. M., Limon, M., Lupton, R. H., Marriage, T. A., Martocci, K. L., Moseley, S. H., Netterfield, C. B., Niemack, M. D., Nolta, M. R., Page, L., Parker, L. P., Reid, B. A., Reintsema, C. D., Sederberg, A. J., Sievers, J. L., Spergel, D. N., Staggs, S. T., Stryzak, O. R., Swetz, D. S., Thornton, R. J., Wollack, E. J., and Zhao, Y., "Systems and control software for the Atacama Cosmology Telescope," in [Proc. SPIE], Bridger, A. and Radziwill, N. M., eds., Advanced Software and Control for Astronomy II 7019, 70192L, SPIE (2008).

[68] Hincks, A. D., Ade, P. A. R., Allen, C., Amiri, M., Appel, J. W., Battistelli, E. S., Burger, B., Chervenak, J. A., Dahlen, A. J., Denny, S., Devlin, M. J., Dicker, S. R., Doriese, W. B., Dünner, R., Essinger-Hileman, T., Fisher, R. P., Fowler, J. W., Halpern, M., Hargrave, P. C., Hasselfield, M., Hilton, G. C., Irwin, K. D., Jarosik, N., Kaul, M., Klein, J., Lau, J. M., Limon, M., Lupton, R. H., Marriage, T. A., Martocci, K. L., Mauskopf, P., Moseley, S. H., Netterfield, C. B., Niemack, M. D., Nolta, M. R., Page, L., Parker, L. P., Sederberg, A. J., Staggs, S. T., Stryzak, O. R., Swetz, D. S., Switzer, E. R., Thornton, R. J., Tucker, C., Wollack, E. J., and Zhao, Y., "The effects of the mechanical performance and alignment of the Atacama Cosmology Telescope on the sensitivity of microwave observations," in [Proc. SPIE], Duncan, W. D., Holland, W. S., Withington, S., and Zmuidzinas, J., eds., Millimeter and Submillimeter Detectors and Instrumentation for Astronomy IV 7020, 70201P, SPIE (2008).

[69] Bennett, C. L. et al., "The microwave anisotropy probe (map) mission," Astrophys. J. 583, 1-23 (2003).

[70] Tegmark, M., "CMB Mapping Experiments: A Designer's Guide," Phys. Rev. D 56, 4514-4529 (1997).

[71] Ade, P. A. R., Pisano, G., Tucker, C., and Weaver, S., "A review of metal mesh filters," in [Society of Photo-Optical Instrumentation Engineers (SPIE) Conference Series], Presented at the Society of PhotoOptical Instrumentation Engineers (SPIE) Conference 6275 (July 2006).

[72] Lau, J., Fowler, J., Marriage, T., Page, L., Leong, J., Wishnow, E., Henry, R., Wollack, E., Halpern, M., Marsden, D., and Marsden, G., "Millimeter-wave antireflection coating for cryogenic silicon lenses," Appl. Opt. 45, 3746 (2006).

[73] Britton, J. W., Niemack, M. D., Beall, J. A., Becker, D., Cho, H. M., Hilton, G. C., Irwin, K. D., and Yoon, K. W., "High-efficiency anti-reflection-coated cryogenic silicon lenses for millimeter wavelengths," in [Proc. SPIE], Holland, W. S. and Zmuidzinas, J., eds., Millimeter and Submillimeter Detectors and Instrumentation for Astronomy V 7741, SPIE (2010).

[74] Yoon, K. W., Appel, J. W., Austermann, J. E., Beall, J. A., Becker, D., Benson, B. A., Bleem, L. E., Britton, J., Chang, C. L., Carlstrom, J. E., Cho, H., Crites, A. T., Essinger-Hileman, T., Everett, W., Halverson, N. W., Henning, J. W., Hilton, G. C., Irwin, K. D., McMahon, J., Mehl, J., Meyer, S. S., Moseley, S., Niemack, M. D., Parker, L. P., Simon, S. M., Staggs, S. T., U-Yen, K., Visnjic, C., Wollack, E., and Zhao, Y., "Feedhorn-Coupled TES Polarimeters for Next-Generation CMB Instruments," in [American Institute of Physics Conference Series], B. Young, B. Cabrera, \& A. Miller, ed., American Institute of Physics Conference Series 1185, 515-518 (Dec. 2009). 
[75] Appel, J. W., Austermann, J. E., Beall, J. A., Becker, D., Benson, B. A., Bleem, L. E., Britton, J., Chang, C. L., Carlstrom, J. E., Cho, H. M., Crites, A. T., Essinger-Hileman, T., Everett, W., Halverson, N. W., Henning, J. W., Hilton, G. C., Irwin, K. D., McMahon, J., Mehl, J., Meyer, S. S., Niemack, M. D., Parker, L. P., Simon, S. M., Staggs, S. T., Visnjic, C., Yoon, K. W., and Zhao, Y., "Characterizing and Modeling the Noise and Complex Impedance of Feedhorn-Coupled TES Polarimeters," in [American Institute of Physics Conference Series], B. Young, B. Cabrera, \& A. Miller, ed., American Institute of Physics Conference Series 1185, 211-214 (Dec. 2009).

[76] Austermann, J. E., Niemack, M. D., Appel, J. W., Beall, J. A., Becker, D., Bennett, D. A., Benson, B. A., Bleem, L. E., Britton, J., Carlstrom, J. E., Chang, C. L., Cho, H. M., Crites, A. T., Essinger-Hileman, T., Everett, W., Halverson, N. W., Henning, J. W., Hilton, G. C., Irwin, K. D., McMahon, J., Mehl, J., Meyer, S. S., Parker, L. P., Simon, S. M., Staggs, S. T., Ullom, J. N., Visnjic, C., Yoon, K. W., and Zhao, Y., "Measurements of Bolometer Uniformity for Feedhorn Coupled TES Polarimeters," in [American Institute of Physics Conference Series], B. Young, B. Cabrera, \& A. Miller, ed., American Institute of Physics Conference Series 1185, 498-501 (Dec. 2009).

[77] Bleem, L. E., Appel, J. W., Austermann, J. E., Beall, J. A., Becker, D. T., Benson, B. A., Britton, J., Carlstrom, J. E., Chang, C. L., Cho, H. M., Crites, A. T., Essinger-Hileman, T., Everett, W., Halverson, N. W., Henning, J. W., Hilton, G. C., Irwin, K. D., McMahon, J., Mehl, J., Meyer, S. S., Niemack, M. D., Parker, L. P., Simon, S. M., Staggs, S. T., Visnjic, C., Yoon, K. W., and Zhao, Y., "Optical properties of Feedhorn-coupled TES polarimeters for CMB polarimetry," in [American Institute of Physics Conference Series], B. Young, B. Cabrera, \& A. Miller, ed., American Institute of Physics Conference Series 1185, 479-482 (Dec. 2009).

[78] McMahon, J., Appel, J. W., Austermann, J. E., Beall, J. A., Becker, D., Benson, B. A., Bleem, L. E., Britton, J., Chang, C. L., Carlstrom, J. E., Cho, H. M., Crites, A. T., Essinger-Hileman, T., Everett, W., Halverson, N. W., Henning, J. W., Hilton, G. C., Irwin, K. D., Mehl, J., Meyer, S. S., Mossley, S., Niemack, M. D., Parker, L. P., Simon, S. M., Staggs, S. T., Visnjic, C., Wollack, E., U.-Yen, K., Yoon, K. W., and Zhao, Y., "Planar Orthomode Transducers for Feedhorn-coupled TES Polarimeters," in [American Institute of Physics Conference Series], B. Young, B. Cabrera, \& A. Miller, ed., American Institute of Physics Conference Series 1185, 490-493 (Dec. 2009).

[79] Essinger-Hileman, T., Appel, J. W., Beal, J. A., Cho, H. M., Fowler, J., Halpern, M., Hasselfield, M., Irwin, K. D., Marriage, T. A., Niemack, M. D., Page, L., Parker, L. P., Pufu, S., Staggs, S. T., Stryzak, O., Visnjic, C., Yoon, K. W., and Zhao, Y., "The Atacama B-Mode Search: CMB Polarimetry with TransitionEdge-Sensor Bolometers," in [American Institute of Physics Conference Series], B. Young, B. Cabrera, \& A. Miller, ed., American Institute of Physics Conference Series 1185, 494-497 (Dec. 2009).

[80] McMahon, J. J., Aird, K. A., Benson, B. A., Bleem, L. E., Britton, J., Carlstrom, J. E., Chang, C. L., Cho, H. S., de Haan, T., Crawford, T. M., Crites, A. T., Datesman, A., Dobbs, M. A., Everett, W., Halverson, N. W., Holder, G. P., Holzapfel, W. L., Hrubes, D., Irwin, K. D., Joy, M., Keisler, R., Lanting, T. M., Lee, A. T., Leitch, E. M., Loehr, A., Lueker, M., Mehl, J., Meyer, S. S., Mohr, J. J., Montroy, T. E., Niemack, M. D., Ngeow, C. C., Novosad, V., Padin, S., Plagge, T., Pryke, C., Reichardt, C., Ruhl, J. E., Schaffer, K. K., Shaw, L., Shirokoff, E., Spieler, H. G., Stadler, B., Stark, A. A., Staniszewski, Z., Vanderlinde, K., Vieira, J. D., Wang, G., Williamson, R., Yefremenko, V., Yoon, K. W., Zhan, O., and Zenteno, A., "SPTpol: an instrument for CMB polarization," in [American Institute of Physics Conference Series], B. Young, B. Cabrera, \& A. Miller, ed., American Institute of Physics Conference Series 1185, 511-514 (Dec. 2009).

[81] Britton, J., Yoon, K. W., Beall, J. A., Becker, D., Cho, H. M., Hilton, G. C., Niemack, M. D., and Irwin, K. D., "Progress Toward Corrugated Feed Horn Arrays in Silicon," in [American Institute of Physics Conference Series], B. Young, B. Cabrera, \& A. Miller, ed., American Institute of Physics Conference Series 1185, 375-378 (Dec. 2009).

[82] Britton, J. W., Yoon, K. W., Beall, J. A., Becker, D., Cho, H. M., Hilton, G. C., Niemack, M. D., and Irwin, K. D., "Corrugated feed horn arrays in silicon," in [Proc. SPIE], Holland, W. S. and Zmuidzinas, J., eds., Millimeter and Submillimeter Detectors and Instrumentation for Astronomy V 7741, SPIE (2010).

[83] Irwin, K. D., "An application of electrothermal feedback for high resolution cryogenic particle detection," Applied Physics Letters 66, 1998-2000 (Apr. 1995). 
[84] Lee, A. T., Richards, P. L., Nam, S. W., Cabrera, B., and Irwin, K. D., "A superconducting bolometer with strong electrothermal feedback," Applied Physics Letters 69, 1801-1803 (Sept. 1996).

[85] Chervenak, J. A., Irwin, K. D., Grossman, E. N., Martinis, J. M., Reintsema, C. D., and Huber, M. E., "Superconducting multiplexer for arrays of transition edge sensors," Applied Physics Letters 74, 4043 (June 1999).

[86] de Korte, P. A. J., Beyer, J., Deiker, S., Hilton, G. C., Irwin, K. D., Macintosh, M., Nam, S. W., Reintsema, C. D., Vale, L. R., and Huber, M. E., "Time-division superconducting quantum interference device multiplexer for transition-edge sensors," Review of Scientific Instruments 74, 3807-3815 (Aug. 2003).

[87] Battistelli, E. S., Amiri, M., Burger, B., Halpern, M., Knotek, S., Ellis, M., Gao, X., Kelly, D., Macintosh, M., Irwin, K., and Reintsema, C., "Functional Description of Read-out Electronics for Time-Domain Multiplexed Bolometers for Millimeter and Sub-millimeter Astronomy," Journal of Low Temperature Physics 151, 908-914 (May 2008).

[88] Yoon, K. W., Appel, J. W., Austermann, J. E., Beall, J. A., Becker, D., Benson, B. A., Bleem, L. E., Britton, J., Chang, C. L., Carlstrom, J. E., Cho, H., Crites, A. T., Essinger-Hileman, T., Everett, W., Halverson, N. W., Henning, J. W., Hilton, G. C., Irwin, K. D., McMahon, J., Mehl, J., Meyer, S. S., Moseley, S., Niemack, M. D., Parker, L. P., Simon, S. M., Staggs, S. T., U-Yen, K., Visnjic, C., Wollack, E., and Zhao, Y., "Feedhorn-coupled TES polarimeters for next-generation CMB instruments," in [Proc. SPIE], Holland, W. S. and Zmuidzinas, J., eds., Millimeter and Submillimeter Detectors and Instrumentation for Astronomy $V$ 7741, SPIE (2010).

[89] Zmuidzinas, J., "Thermal noise and correlations in photon detection," Applied Optics 42, 4989-5008 (Sept. 2003).

[90] Niemack, M. D., "Towards Dark Energy: Design, Development, and Preliminary Data from ACT," Ph.D. Thesis, Princeton University (2008). 